\author{
A Ground Systems Architecture Transition for \\ a Distributed Operations System \\ Donna Sellers, NASA, Marshall Space Flight Center \\ Lee Pitts, Lockheed Martin, Marshall Space Flight Center \\ Barry Bryant, Lockheed Martin, Marshall Space Flight Center
}

\begin{abstract}
The Marshall Space Flight Center (MSFC) Ground Systems Department (GSD) recently undertook an architecture change in the product line that serves the ISS program. As a result, the architecture tradeoffs between data system product lines that serve remote users versus those that serve control center flight control teams were explored extensively. This paper describes the resulting architecture that will be used in the International Space Station (ISS) payloads program, and the resulting functional breakdown of the products that support this architecture. It also describes the lessons learned from the path that was followed, as a migration of products cause the need to reevaluate the allocation of functions across the architecture.
\end{abstract}

The result is a set of innovative ground system solutions that is scalable so it can support facilities of wide-ranging sizes, from a small site up to large control centers. Effective use of system automation, custom components, design optimization for data management, data storage, data transmissions, and advanced local and wide area networking architectures, plus the effective use of Commercial-Off-The-Shelf (COTS) products, provides flexible Remote Ground System options that can be tailored to the needs of each user. This paper offers a description of the efficiency and effectiveness of the Ground Systems architectural options that have been implemented, and includes successful implementation examples and lessons learned.

\title{
Introduction and Background
}

The MSFC GSD is responsible for the development and operation of the Payload Operations Integration Center (POIC) at the Huntsville Operations Support Center (HOSC). The POIC is an International Space Station (ISS) facility that manages on-orbit ISS payloads and payload support systems in coordination with the Mission Control Center in Houston (MCC-H), the distributed International Partner (IP) Payload Control Centers (PCCs), Telescience Support Centers (TSCs), and payload-unique facilities. Figure 1 shows the POIC Payload Operations flow with the ISS. 


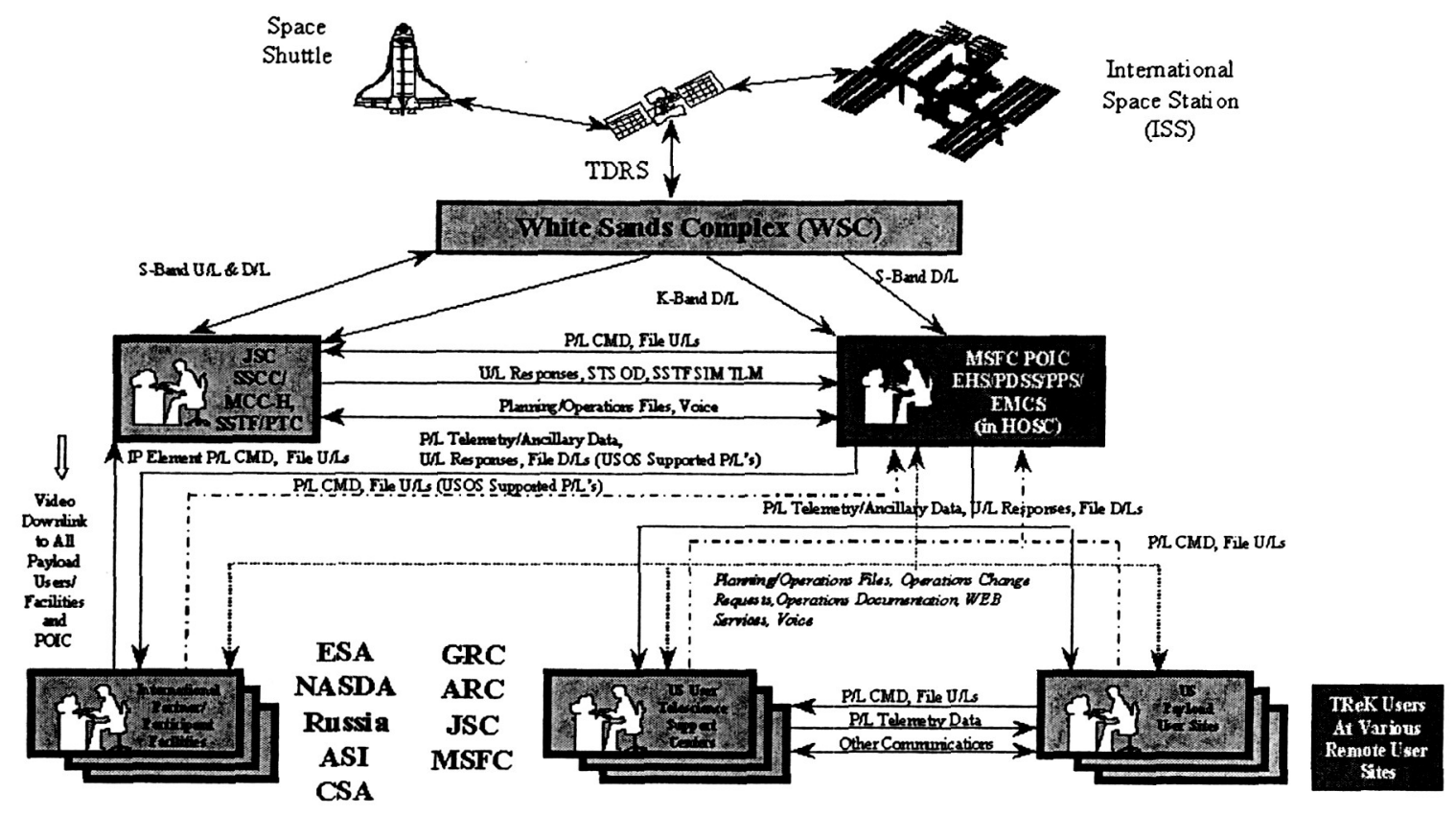

Figure 1 ISS Payload Operations with the POIC

The POIC is a complete operations facility composed of a ground system responsible for distributing data, voice, and video for payload science operation. The POIC is comprised of four primary systems:

1) The Enhanced Huntsville Operation Support Center (HOSC) System (EHS) which performs command processing and real-time and near-real-time telemetry processing for pre-launch integration and checkout, simulation training and flight operations;

2) The Payload Data Services System (PDSS), which acquires, stores, and distributes ISS data to the EHS System, IPs, TSCs, and other payload-unique facilities;

3) The Enhanced Mission Communications System (EMCS), which receives voice, video and data from external interfaces and distributes voice, video and data to the wide area network service providers for distribution to remote users.

4) The Payload Planning System (PPS) which provides a set of software tools to automate planning and schedule payload activities;

ISS remote operations with the POIC have been primarily accomplished in three ways.

1) Users remain at home site and obtain a copy of Telescience Resource Kit (TReK) software and associated Commercial Off the Shelf (COTS) software to provide their telemetry and commanding capabilities. Note: TReK uses X-windows and Web software to access other required POIC capabilities.

2) Users remain at home site and develop software for Ground Support Equipment (GSE) to utilize POIC telemetry and command interfaces and use an X-windows and or Web session to access required POIC capabilities. 
3) Users remain at home site and use an X-windows and/or Web session to access required POIC capabilities. Note: Users need to provide their own TReK or GSE workstations to process telemetry as the POIC offers only limited telemetry processing.

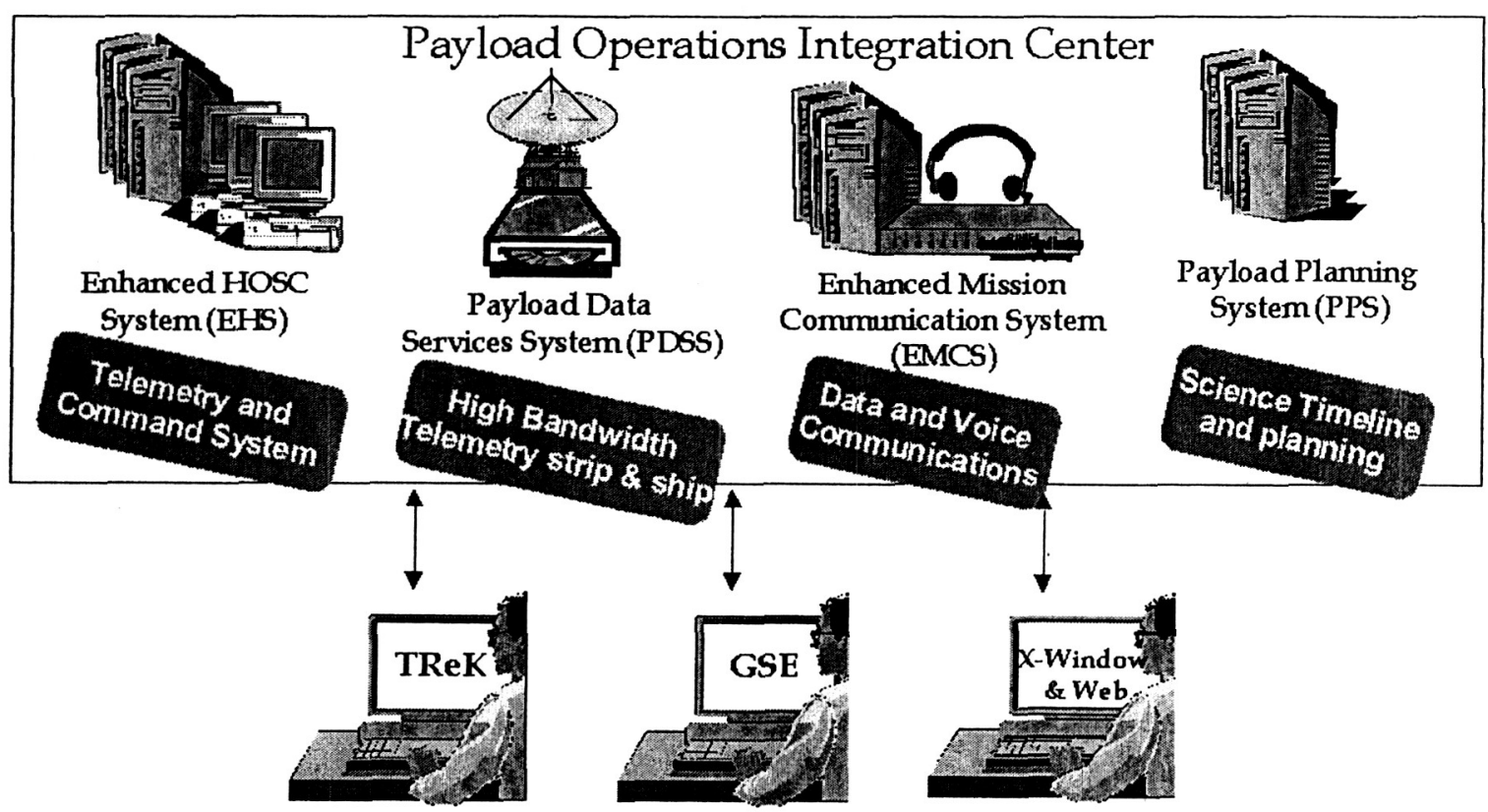

Figure 2 POIC and Remote Systems

The EHS system is currently supported at two remote sites:

1) A separate operational EHS baseline is maintained at the Chandra (CXO) Operations Control Center in Cambridge, Massachusetts. Resources are shared with the ISS program but a separate contract maintains the CXO baseline.

2) A complete copy of EHS is operational at the ISS Payload Test and Checkout System (PTCS) at Kennedy Space Center (KSC). The PTCS uses the EHS software to checkout payload interfaces prior to launch. The EHS at the PCTS is the same baseline system as the EHS system operational at the POIC.

\section{EHS Initial Development and Philosophy}

The POIC went into operation in support of the ISS in February of 2001 after an eight (8) year development and deployment activity. This included transitioning from a successful Spacelab and Space Transportation System (STS) mission support environment based on minicomputer architecture to a traditional client server model. In addition, the concept of users dispersed over a broad geographical area, including IP's was required. These partners around the globe would have to be supported by United States export restrictions and require both open standards and utilization of products that are widely available.

During initial design and development phases the philosophy was to design the best system in support of these ISS requirements. Successful past support of mission environments led to a traditional client server model being selected for the POIC systems. The architecture design was based on what technology was available to support an integrated system that met open standards 
and was portable across the globe. At the time the only thing available that would support these requirements were high cost server/client solutions. The MSFC GSD made a decision to use UNIX based servers and workstations that provide a secure integrated solution. This high cost solution and development effort drove budget limits based on design and needs. The direction was moving to "design the requirements."

\section{Changing Philosophy Forces Architecture Tradeoffs and Lessons Learned}

When the ISS reached an operational state several problems began to surface. The ISS budget was becoming severely constrained. Strong inputs from ISS users such as Telescience Support Centers (TSCs) and International Partners (IPs) suggested that high costs solutions were no longer an option. With most of the requirements development complete, many of the POIC hardware components were reaching their end of life, COTS were limited in capability for expensive systems forcing COTS product costs to soar and hardware and software maintenance became almost unmanageable. The Enhanced HOSC System (EHS) was operational yet in a couple of years it would be impossible to maintain. New approaches were needed to reduce the cost of refurbishment, maintenance, and portability of the POIC. The philosophy was changing to a "design by force."

New approaches were developed or designed to alleviate these problems and to maintain the future of the POIC. Architectural tradeoffs were needed along with knowledge based on lessons learned. Dramatic reductions in cost of operations, maintenance, refurbishment, and deployment of a ground system were realized. Several specific initiatives were begun, to move to commodity low-cost computers, low-cost communications schemes, and replace high cost licensed COTS software. These initiatives and reductions lead to a broad range of deployment options to include secure remote PCs, highly scalable and extensible ground systems with minimal initial investment, and the capability to fully support institutional users with a total ground system. The basic premise underlying each study is that the replacement of hardware and software components with commodity based items wherever applicable will dramatically reduce the overall lifecycle cost of the project. These initiatives are discussed in the following sections.

\section{Replace EHS Client Workstations}

The first initiative leveraged several lessons learned from existing POIC remote payload operations on a lower cost Windows ${ }^{\mathrm{TM}}$ based system. First, Remote ISS payload users were using the PC-based Telescience Resource Kit (TReK), which provided a stand-alone command and telemetry system on a Windows NT/2000 PC significantly improving payload customer command, control, and science data processing. Remote users were using an Intel PC to basically do remote operations securely and efficiently. The first lesson learned was that high-end UNIX systems are not needed to do remote operations. Secondly, Remote ISS users were using the secure, three-tier, web architecture and the X-Window interface to the EHS UNIX servers for commanding and access to POIC applications. Windows ${ }^{\mathrm{TM}} \mathrm{Web}$ and $\mathrm{X}$-Windows was proven to be a stable client platform for remote ISS users and provided strong and affordable solutions. Remote ISS users were almost doing the same job with Web and X-Windows as the POIC workstations.

MSFC GSD began investigating whether a Windows system could support the same capability that the POIC Workstations were supporting. Technology for Intel PCs and Windows ${ }^{\mathrm{TM}}$ had change considerably providing both a diverse development environment and the necessary security and control needed by the POIC. The X-Windows protocol and Web-based services standards were defined for local and remote users in the HOSC Common User Interface Standard 
(MSFC-STD-1956) for EHS X-Windows-based applications and PC applications using Microsoft standards. The workstations were basically using X-Window and Web technology in the POIC with some local operation. This concept would lend itself to the migration to a PC. A solution was to migrate some of the $\mathrm{X}$-intensive applications to a Windows ${ }^{\mathrm{TM}}$ environment and provide an integrated X-Window interface to other POIC server applications. This integration between local running $\mathrm{PC}$ applications and the $\mathrm{X}$-Window interface POIC server applications would be transparent to the user. Now the POIC could have EHS running on a PC, which became known as EHS PC (EPC).

Again, a strong lesson was to use what was proven to work by the remote ISS users and build upon its strengths while providing cheaper solutions to meet a decreasing budget. The overall solution was to replace UNIX Workstations with lower cost Intel based Windows 2000 PC platforms and migrate EHS X-intensive UNIX applications to local Windows ${ }^{\mathrm{TM}}$ applications. The total cost savings of replacement is estimated to be at least $\$ 1.6$ million through fiscal year 2008 (FY08), a \$2.8 million cost avoidance, from previous POIC budget submissions.
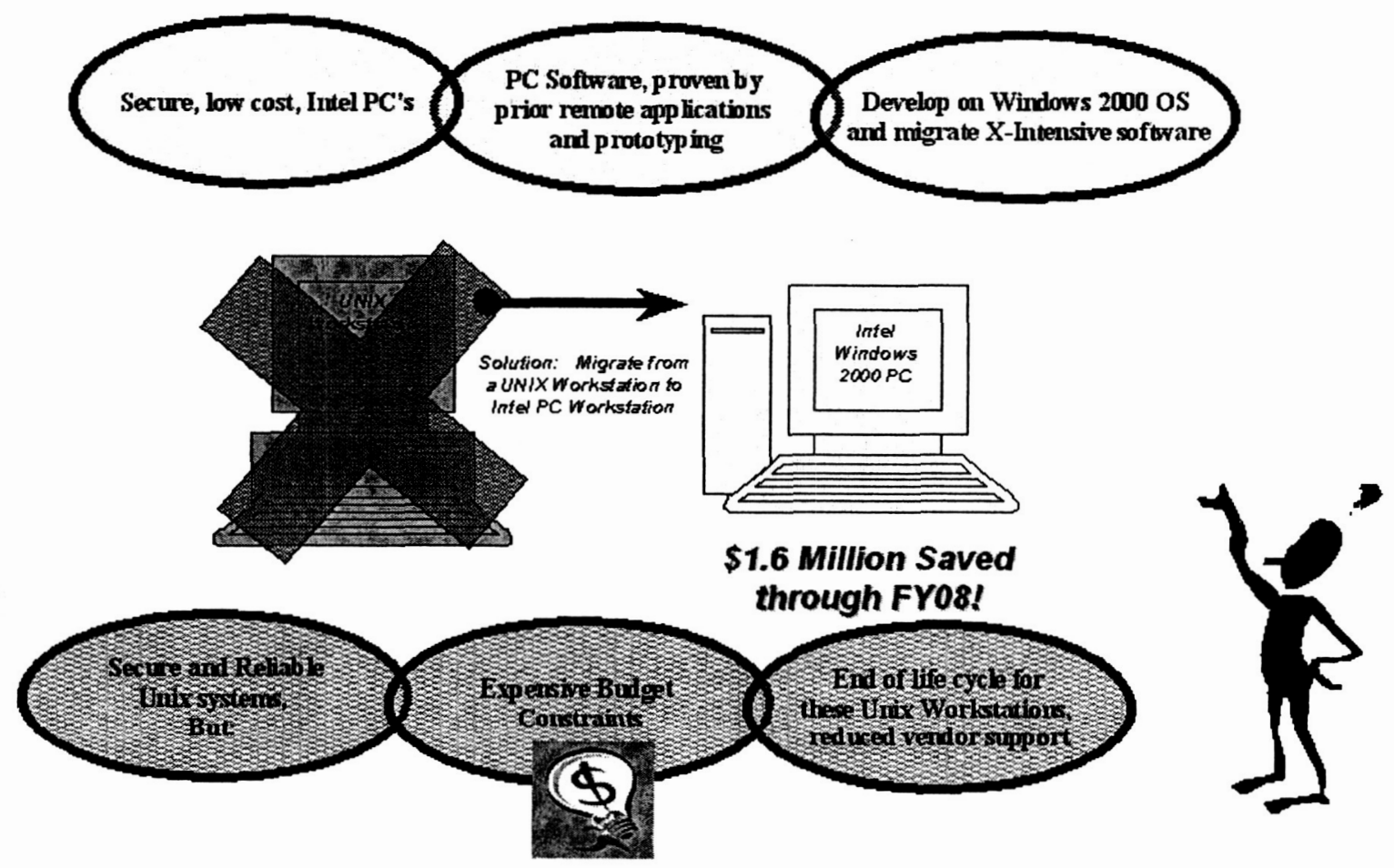

Figure 3 Replace EHS Client Workstations

\section{Review EHS and PDSS Server Architecture}

Another initiative reviewed the server architectures of the PDSS data distribution systems and EHS command and telemetry system and proposed the migration to new platforms for both hardware and software. The current hardware server implementation was nearing the end of its life cycle and the hardware and maintenance costs were soaring. The approach was to find a solution for the high maintenance costs of hardware and provide a more stable long-term solution.

Several factors were a plus in the architecture approaches that were selected. The EHS server applications were developed on and executed in a UNIX operating system environment in order to support multitasking applications with multiple users simultaneously for ISS payload 
commanding and telemetry processing. The applications using $\mathrm{C}$ language were built to the American National Standards Institute (ANSI) C language standards and are Portable Operating Systems Interface (POSIX) compliant. Therefore, if we could find a UNIX based solution, the software should be easily portable. Fortunately, Moore's Law (the computational power available at a particular price doubles every 18 months) and market forces had changed the computing landscape considerably. High-end Intel based PCs were becoming more and more affordable and provided extremely huge computing power with the support of several different stable operating systems. Linux was becoming a major operating system supported by most vendors on Intel based PCs.

Together with the POSIX compliance and the Intel power, Intel PC's and Linux were a definite solution for both the EHS and PDSS server architecture at very low costs. Total cost savings for PDSS redesign is estimated to be at least $\$ 2.6$ million through FY08 from previous POIC budget submissions. EHS redesign is estimated to be at least $\$ 3.9$ million total savings through FY08 with a $\$ 3.6 \mathrm{M}$ cost avoidance from previous POIC budget submissions.

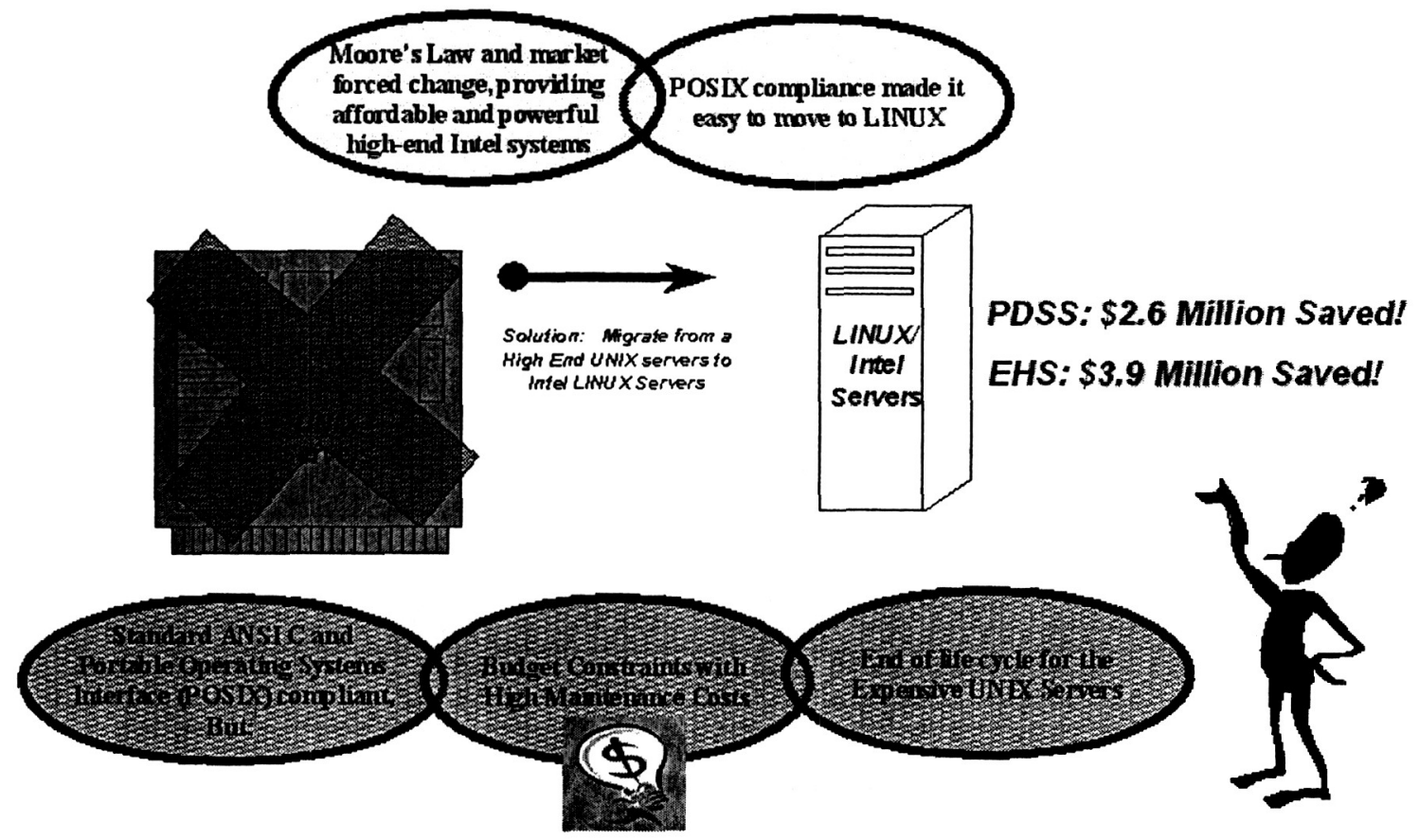

Figure 4 Review EHS and PDSS Server Architecture

\section{Examine EHS COTS Products and Value Added}

A third study initiated a review of COTS products to examine the level of added value of each product by reducing or eliminating dependencies on selected expensive COTS software products. This study included replacement of some COTS products with custom code, deletions, substitutions, and consolidation of COTS products. One of the major COTS simplification and redesign was accomplished with the Payload Information Management System (PIMS). PIMS is a ground-based electronic document configuration management and change management system with an embedded workflow approval process. Architecturally, the PIMS system employs a multi-tiered, web-based architecture with a centralized vault. PIMS developers found that by utilizing pervasive 
standards and technologies, they could maximize platform, system, and facility interoperability while servicing all interfacing needs. PIMS was wrapped around a major "all-in-one" COTS product, which was adapted to the ISS workflow system for payload users to manage and approve changes in the realtime environment. This COTS product was removed and replaced with custom-built code. It was determined that "all-in-one" products should not be used unless operations concepts, processes, requirements, and user interface needs can be easily adapted to conform to the selected tool. Other smaller COTS products, which had no constraints to requirements, were used for the PIMS system. The PIMS redesign significantly reduced the POIC Operations and Maintenance and is estimated to be at least $\$ 3.3$ million total savings minimum through FY08 from previous POIC budget submissions.

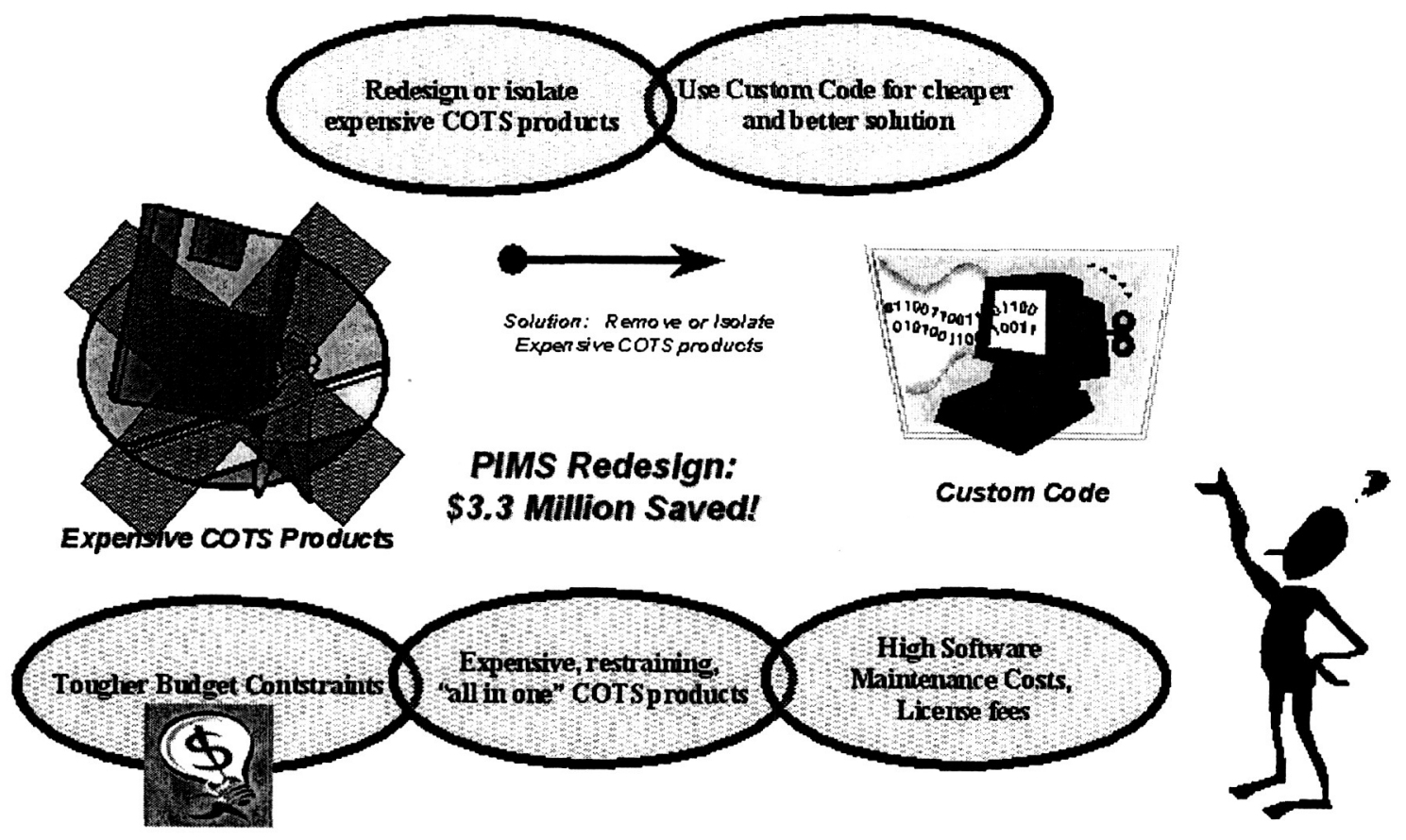

Figure 5 Examine COTS Products and Value added

\section{Security and Technology Advantages}

Security and network technology began to evolve during all these studies and migrate to a more efficient, effective way of doing business. Once the ISS reached an operational state with both local and remote users it networks and security became a very hot topic. Global Operations became a necessity, yet protection of the crew and vehicle systems was still required. The POIC was required to not only manage daily operations but also manage those who were controlling onboard payloads. Due to the POIC's diverse user community, security evolved from an isolated but open system to a system, which supports local and remote access to ISS. This was accomplished through the use of an evolved security strategy, Public Key Infrastructure (PKI), and custom design. The original POIC firewall was not secure enough for an international facility with its old Internet Protocol technology. Based on several studies it was decided to use an integrated firewall with Virtual Private Networks (VPN). 
Fortunately, network standards were evolved and set by initial network design. LAN communications' stack were in compliance with the International Standards Organization (ISO) Open Systems Interconnection (OSI) model with platforms (workstations, servers, and peripherals), and networked to approve standards at the physical, data link, network, and transports layers. Ethernet standards were fully supported at the physical and data link layers, with the IP supporting the network layer. The transport layer supported both the Transmission Control Protocol (TCP) and User Datagram Protocol (UDP) standards. Shared media moved to switched devices at lower cost. An added benefit of Internet Protocol switching was enhanced security on the networks. High bandwidth intercommunications were needed and the use of worldwide science and education networks, such as the Abilene network, became a cheaper and better solution.

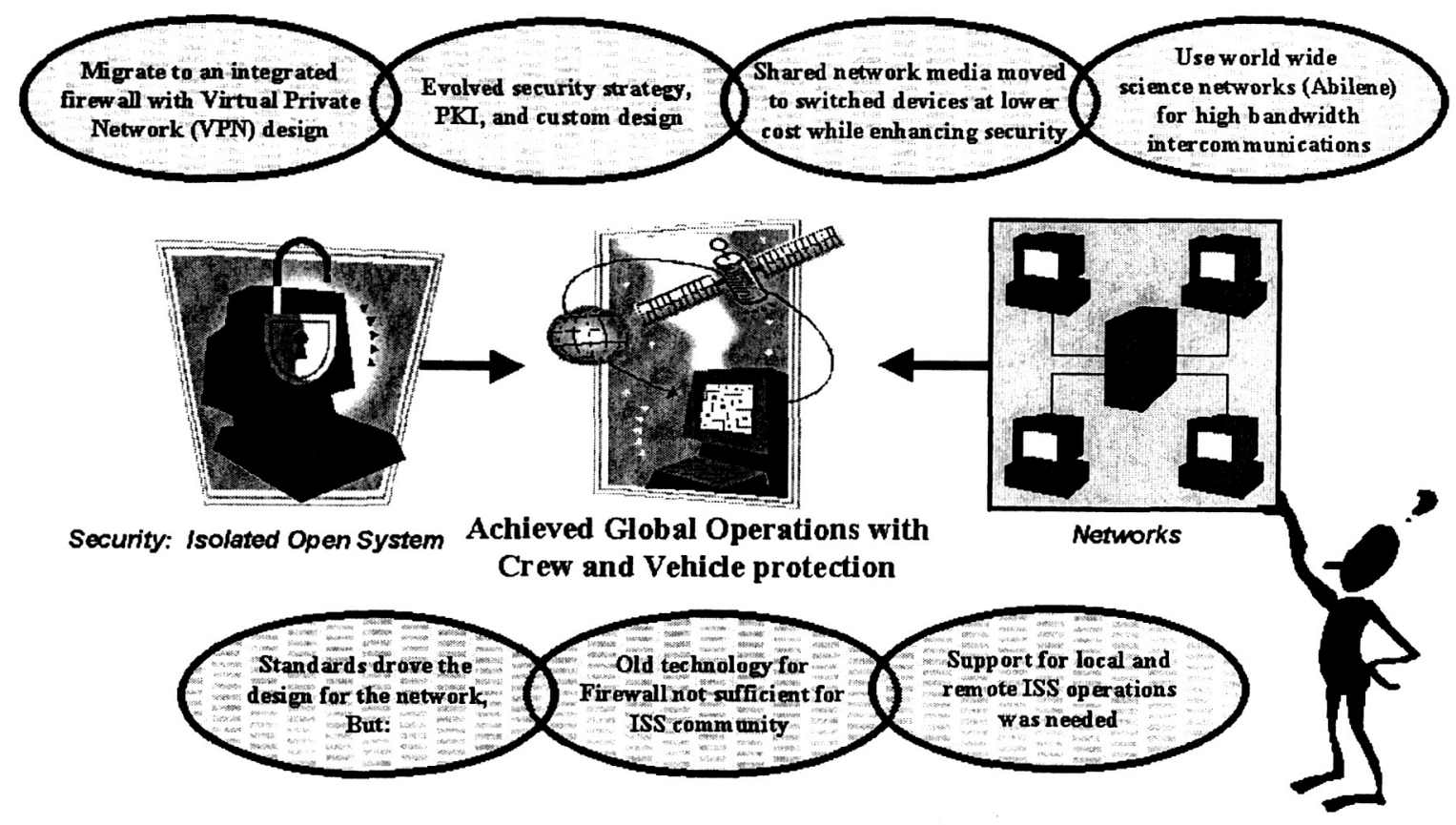

Figure 6 Security and Network Advantages

\section{Voice Over Internet Protocol (VOIP) Technologies}

The final initiative reviewed Voice Over Internet Protocol (VOIP) communication technologies, developed an operational model for flight operations, and demonstrated that voice over the internet was practical and could be integrated into operations. Early remote ISS users received a loaned EMCS keyset and the ISS budget was responsible for leasing a circuit for each Enhanced Voice Operations Distribution System (EVoDS) session. A low cost solution was needed. The loaner solution was too expensive for the ISS program office. Initial development determined that a mission voice system would provide secure mission audio service to remote users via Internet Protocol (IP) based communications using a Windows ${ }^{\mathrm{TM}} 2000$ based personal computer with COTS software, sound card, and headset. This system became known as the Internet Voice Distribution System (IVoDS). It was not only cheaper, but deployment was easier via the Web. No more The need to ship and maintain loaner EVoDs was eliminated. Security was an issue, but security technology had changed with the use of VPNs and science and education networks. The VOIP technology had advanced so much that it was possible to do voice communications with the necessary quality and security needed for ISS communications easier and at a cheaper cost. 


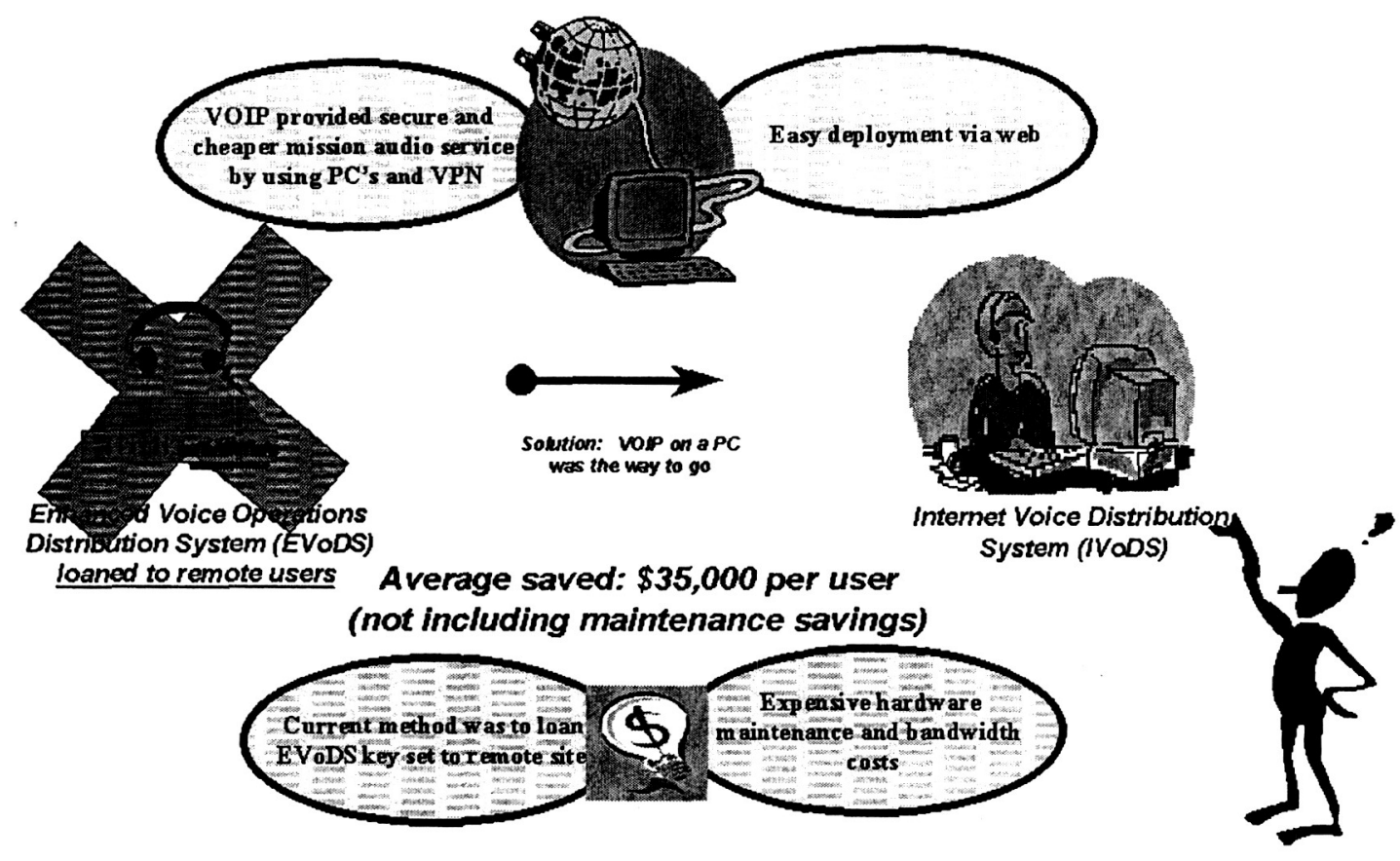

Figure 7 Voice Over Internet Protocol (VOIP) Technologies

\section{Transition Philosophy and Timing}

During the last few years the transition to PC and Intel technology changed significantly. PC and Intel performance hit the threshold that allowed PCs to take on functions previously only possible with workstation-class machines. PC Security controls matured to an acceptable level such that when combined with other measures (isolated networks, firewalls, access controls, etc.) the security risks were acceptable. PCs have saturated the market, providing low cost, easy transition. Linux and Windows ${ }^{\mathrm{TM}}$ operating systems became viable development options. Linux versus UNIX was secure and compliant. Software was widely supported on both Linux and Windows, with lower costs for maintenance

The risks and abatements for the server and PC transition were seriously looked at. One concern was with the project management risk of expensive server code porting. Concern was abated because of historical emphasis on POSIX-compliant code in UNIX workstations. Prototyping initially indicated risk would be low and validated that assessment. Code was recompiled with very few modifications. Another concern was with the PC Migration risk of user disruption during operations, retraining, etc. This methodology was established initially using PCs to Xwindow into UNIX workstations for "Legacy" applications. The first few applications were ported to "Native" on PC. Other applications still ran as Legacy (Server) versions and were available to user simultaneously. User teams verified usability, and transitioned at their pace. It was determined that remaining applications would be ported to "Native" mode as budget and schedule permitted.

Additional benefits were that users, especially new trainees, were more familiar with the PC platform, reducing basic orientation training. The resulting system was more deployable to remote locations at lower cost. The system offered more scalability and could be scaled down or up, now that more functions would fit in fewer boxes. This offers options to small, medium, or 
large payload centers, with higher power applications supported by commodity hardware. The overall architecture allows breaking server functions across multiple server hardware systems.

\section{Architecture Tradeoffs led to Operational Solutions}

High cost hardware and software maintenance drove requirements for more innovative and low cost ways of doing distributed payload operations at the POIC. The architectural changes and tradeoffs led to more scalable, low cost solutions for international secure operations at the POIC. These operational solutions are so streamlined and efficient they are portable enough to use remotely in ISS facilities and by users along with the remote solutions such as TReK that are already available to the remote Payloads to monitor and control their on-orbit experiments. Innovations with the POIC Intel server and Windows ${ }^{\text {TM }} 2000$ PC's solutions plus the IVoDS system could also provide data receipt, processing, storage, post processing analysis, commanding, and voice distribution both locally and remotely. Remote ISS users could now use these solutions such as the EHS PC (EPC) and IVoDS to communicate with the ISS Vehicle. All solutions are scalable and range from POIC interface-compliant clients suitable for small Payload teams to a set of servers and associated clients suited more for large facility outfitting.

\section{Scalable Solutions}

Individual and small payload teams who need to monitor and control individual payloads may be interested in one or more remote solutions. The first solution for Small payload Teams is a suite of software applications called the Telescience Resource Kit (TReK); that provides local ground support system functions for Telemetry and Commanding. TReK is primarily for the Science users who want full control, with local autonomy. TReK provides local database, recording and playback functions as well as an Application Programming Interface (API) for scientist to use their favorite COTS products to build command and telemetry displays and computations. A second solution for Small Payload teams which need more centralized/integrated control is a PC suite of software applications called EHS PC (EPC). EPC combines the use of a built in XWindow interface that allows remote users to access all existing Payload Ops user tools from the POIC, with the added benefit of local PC Windows applications which communicate with the existing POIC services. This suite of software allows the remote POIC users to share products with the POIC and run them locally on the PC, such as telemetry displays that can be used in payload troubleshooting. Remote PC solutions for voice can also be obtained through the Voice over the Internet (VOIP) PC based suite of software called Internet Voice Distribution System (IVoDS). IVoDS allows Internet-based participants to talk/listen on one conference while listening to seven others. This multi-conferencing system links researchers, NASA Operations Personnel, and the ISS on-orbit astronauts together to support Space Station experiment operations \& planning. Additional POIC services that can be used with any of the options described above for Payload Operations are the EHS Web Interfaces which allow for viewing and executing reports from the telemetry and command databases, access for defining/initiating processed telemetry parameter messages (termed Ground Support Equipment (GSE) packets) transmitted to user systems, tracking of uplink commands and post analysis of those commands, as well as information management and planning systems which provide adaptable workflow technologies for operations execution and receipt storage and distribution of documentation and other user products. Depending on the Customer's requirements users may also want to use a combination of TReK, EPC, and IVoDS. 


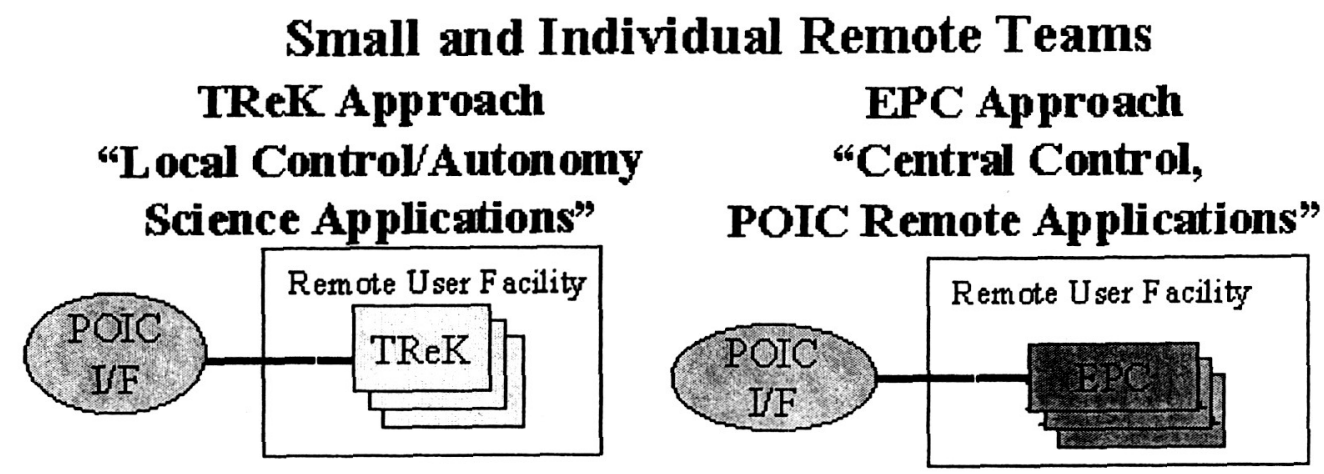

Combination Approach

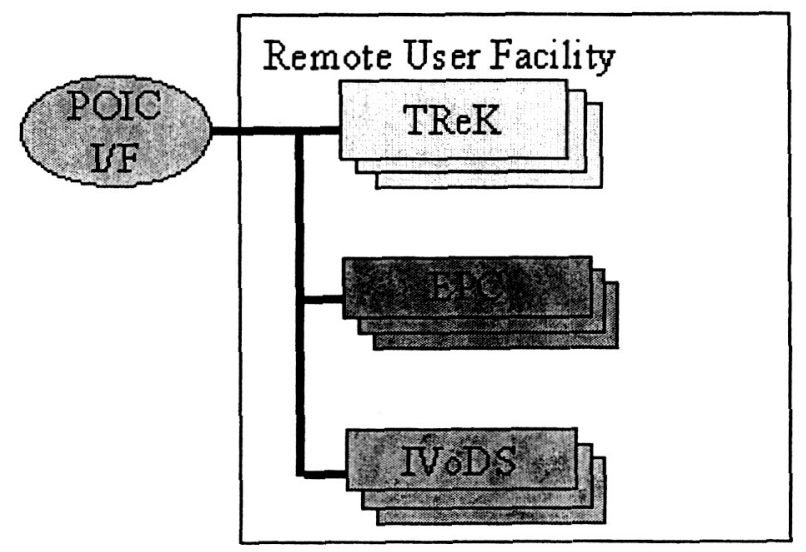

*Note: All options may utilize the EHS Web Interfaces

Medium and large payload teams or control centers can choose both the previous options or choose to implement a copy of the low cost EHS Intel server and PC desktop architecture implemented in the POIC. A copy of the EHS servers allow for scalable and cost effective systems that easily expand from supporting a few local users to supporting many users, local and remote. This option also allows for secure internal and external communications through the use of firewalls and Virtual Private Network (VPN) technology. 


\section{Medium and Large Remote Teams}

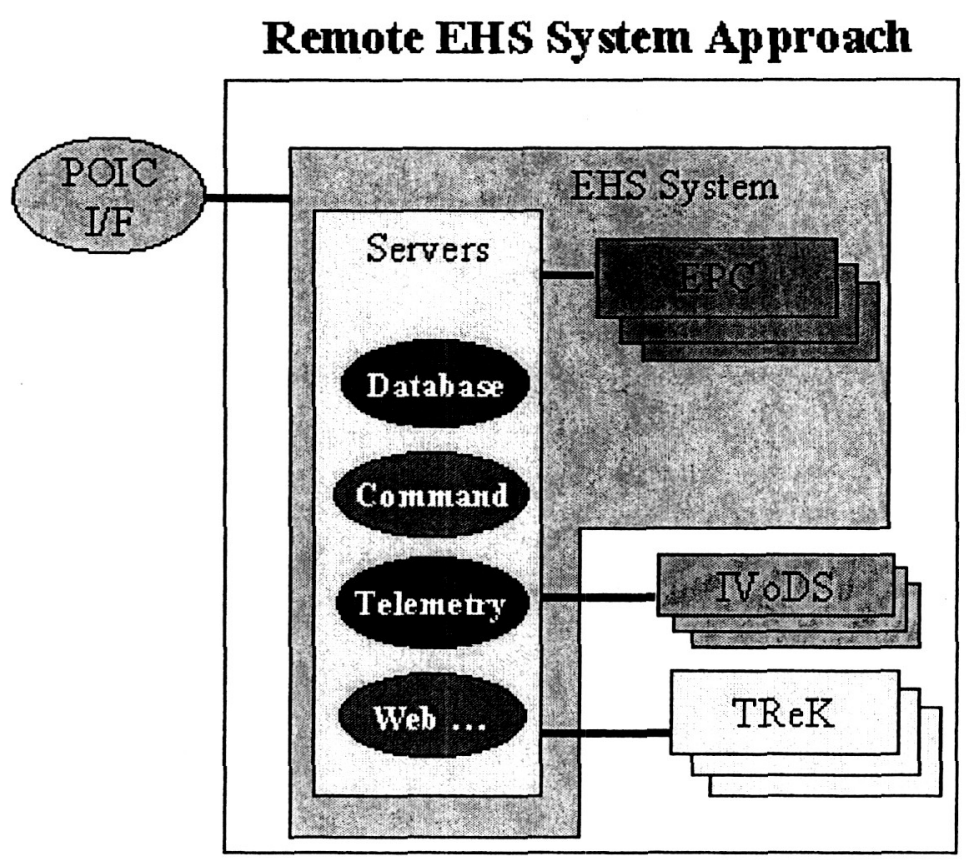

\section{Summary}

The GSD architecture changes began a revolution of innovative solutions, costs savings, and customer satisfaction. The decisions were forced by open market and end of life cycle products that were too expensive to keep the POIC operational. By finding tradeoffs and new ways of doing business the POIC became a new and better facility, operating more efficiently in support of local and remote users. The path that followed has widened to include scalable products that can be focused on customer needs and requirements.

The goal is to continue evaluating products and solutions so that ground systems cannot only be scalable and affordable, but generic across all projects. Technology changes everyday and becomes better, faster, and cheaper which is where MSFC GSD will continue to move.

\section{Special Appreciation Acknowledgement}

The initiatives that are discussed in this paper are a direct result of the hard work and accomplishments of the Ground Systems Department (GSD) team. This team is made up of NASA, the Utilization Mission Systems (UMS) contractor, and other supporting contracts. Without their wonderful accomplishments this paper could not have been written. Thanks to all who have contributed.

\section{References}

NASA Marshall Space Flight Center, HOSC Common User Interface Standard (MSFC-STD1956) 
NASA Marshall Space Flight Center, POIC Capabilities Document, NASA Document SSP50304, Rev. A, October 1999.

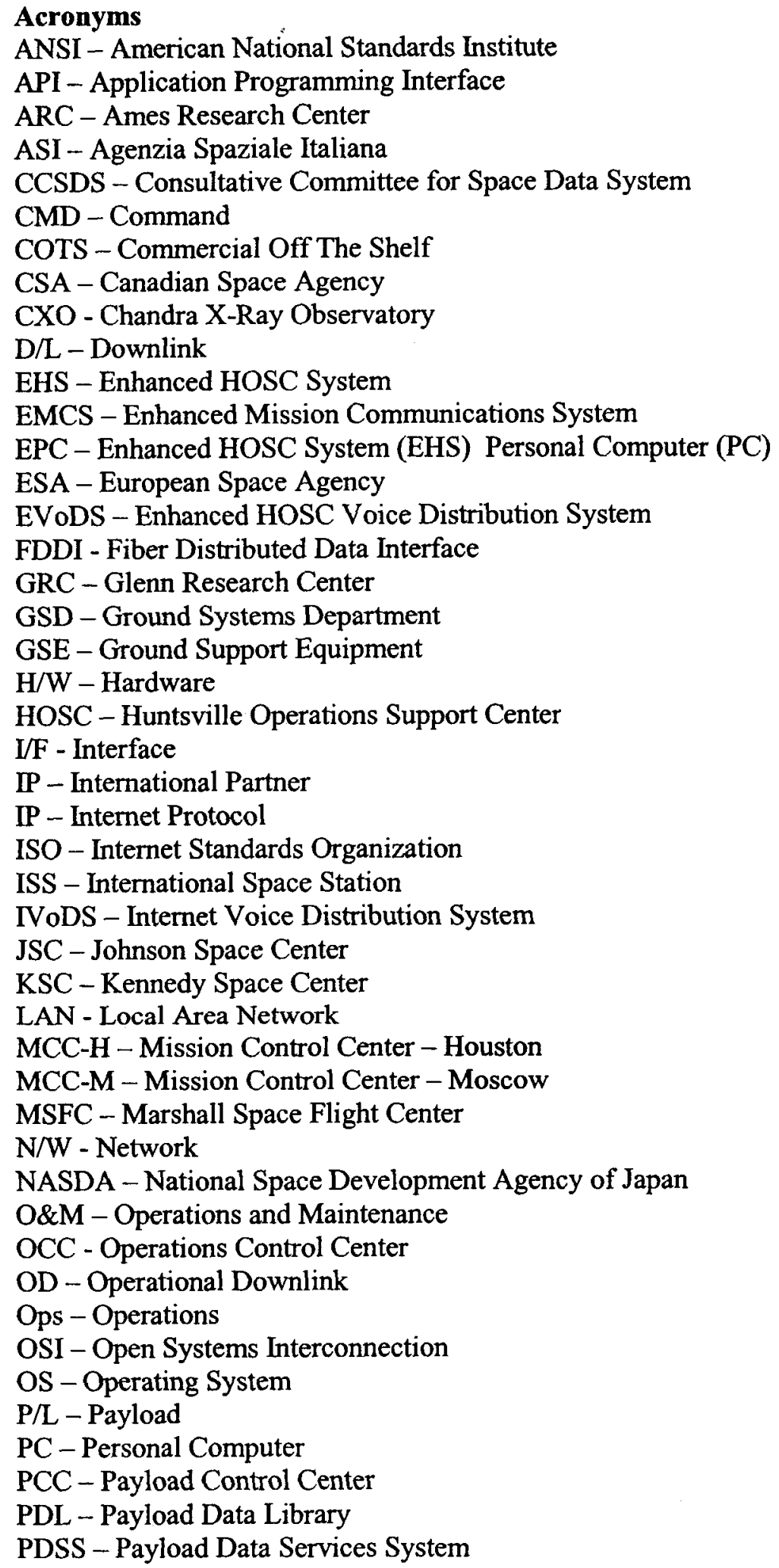


PIMS - Payload Information Management System

PKI - Public Key Infrastructure

POIC - Payload Operations Integration Center

POSIX - Portable Operating System Interface (UNIX)

PPS - Payload Planning System

PSIV - Payload Software Integration and Verification

PTC - Payload Training Capability

PTCS - Payload Test and Checkout System

S/W - Software

SIM - Simulation

SL - Spacelab

SSCC - Space Station Control Center

SSTF - Space Station Training Facility

STS - Space Transportation System

TCP - Transmission Control Protocol

TDRS - Tracking And Data Relay Satellite

TLM - Telemetry

TReK - Telescience Resource Kit

TSC - Telescience Support Center

U/L - Uplink

UDP - User Datagram Protocol

UDSM - User Data Summary Message

UMS - Utilization Mission Systems

USOC - United States Operations Center

USOS - United States On-Orbit System

VOIP - Voice Over The Internet

VPN - Virtual Private Network

W/S - Workstation

WSC - White Sands Complex

$\mathrm{X}-\mathrm{X}$-Windows

XPG - X-Open Portability Guide 


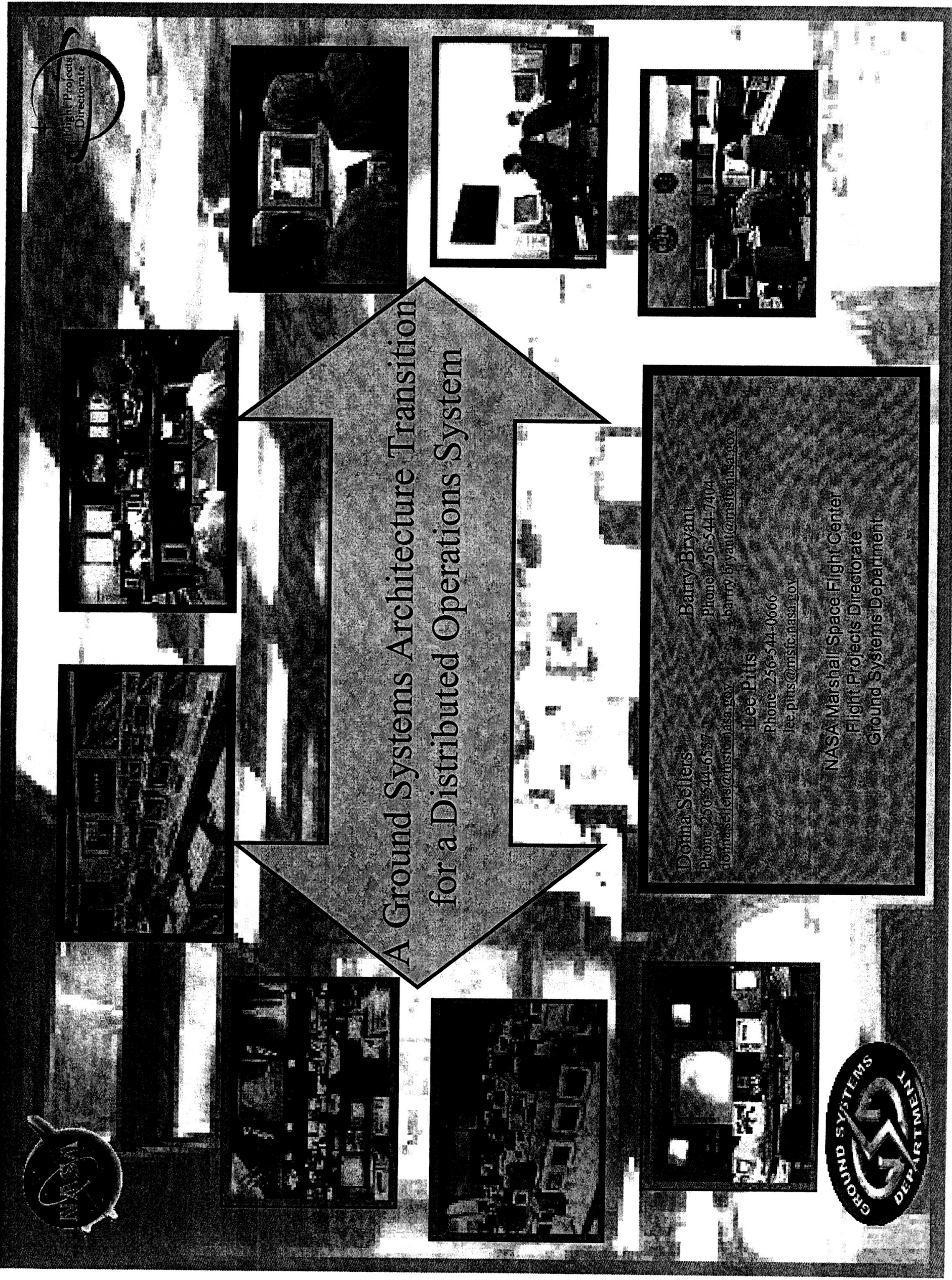




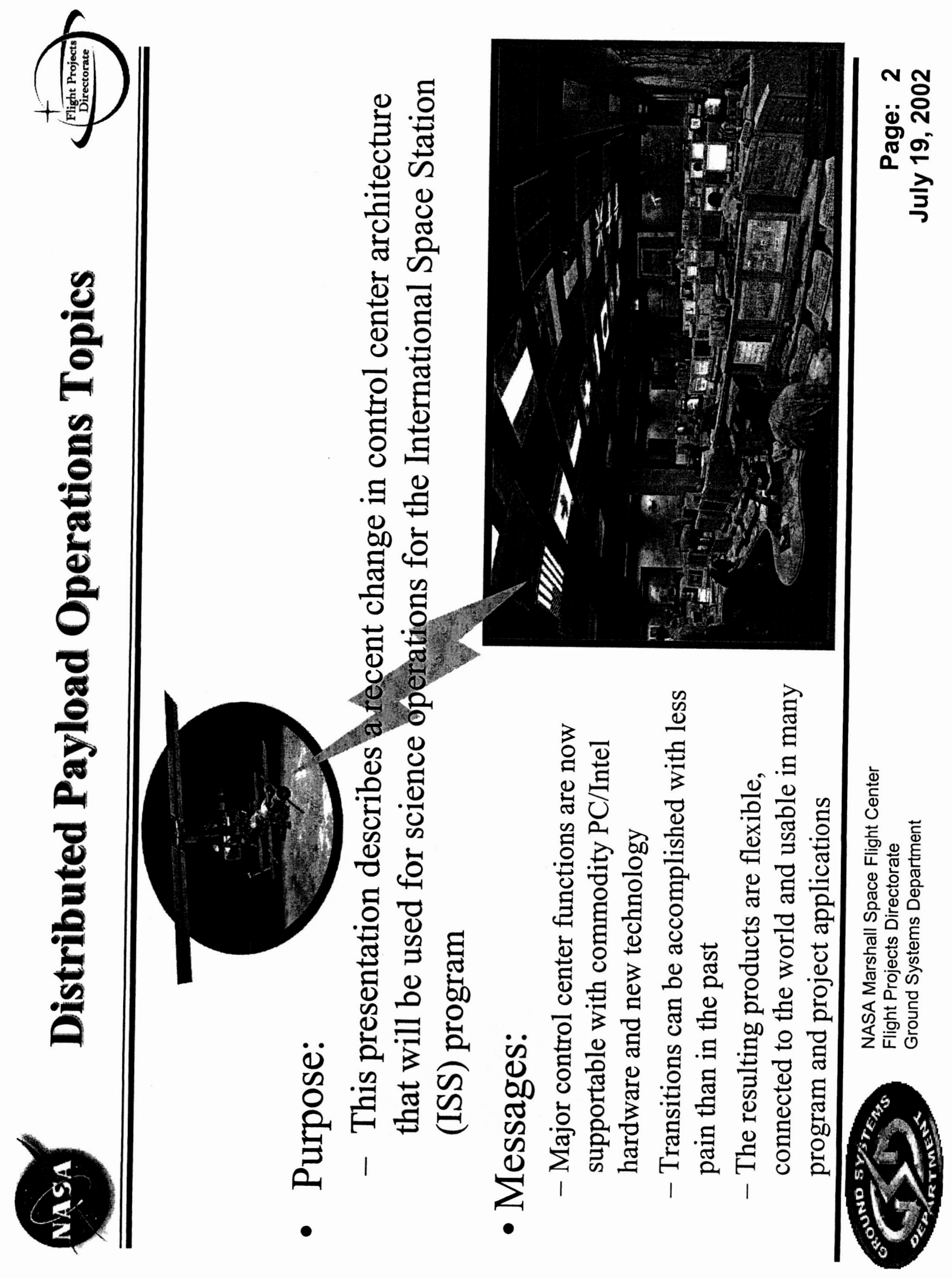




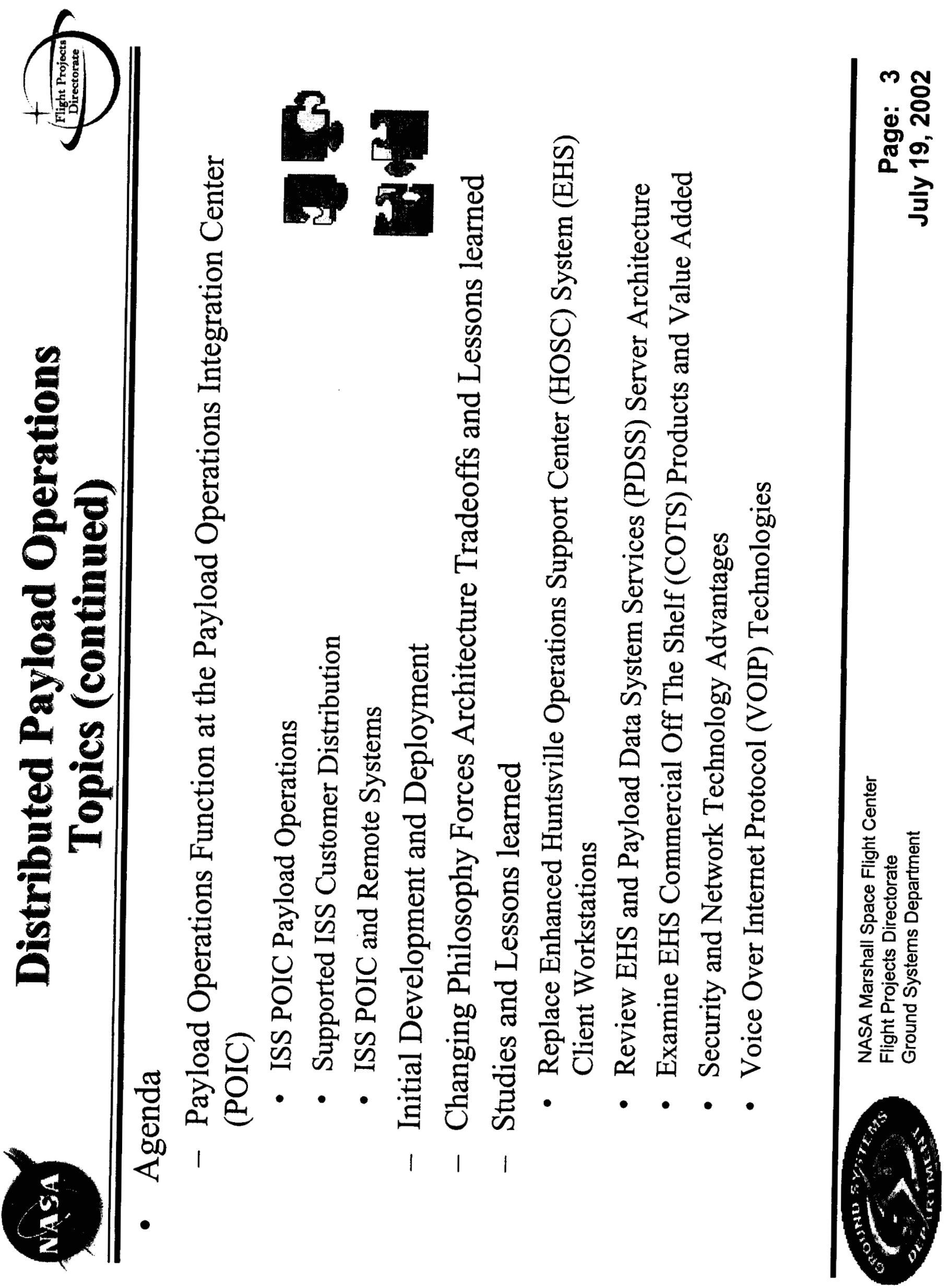




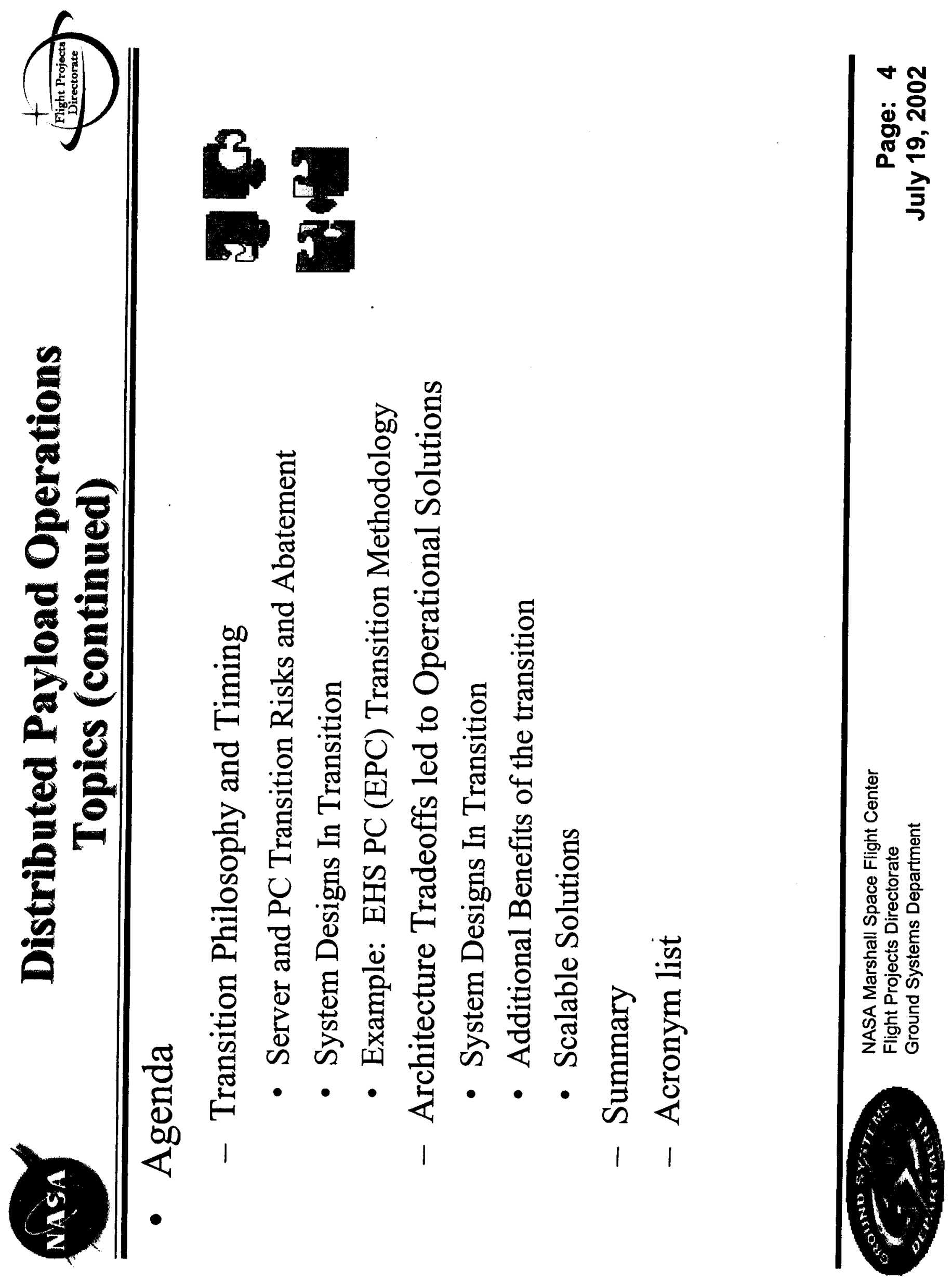




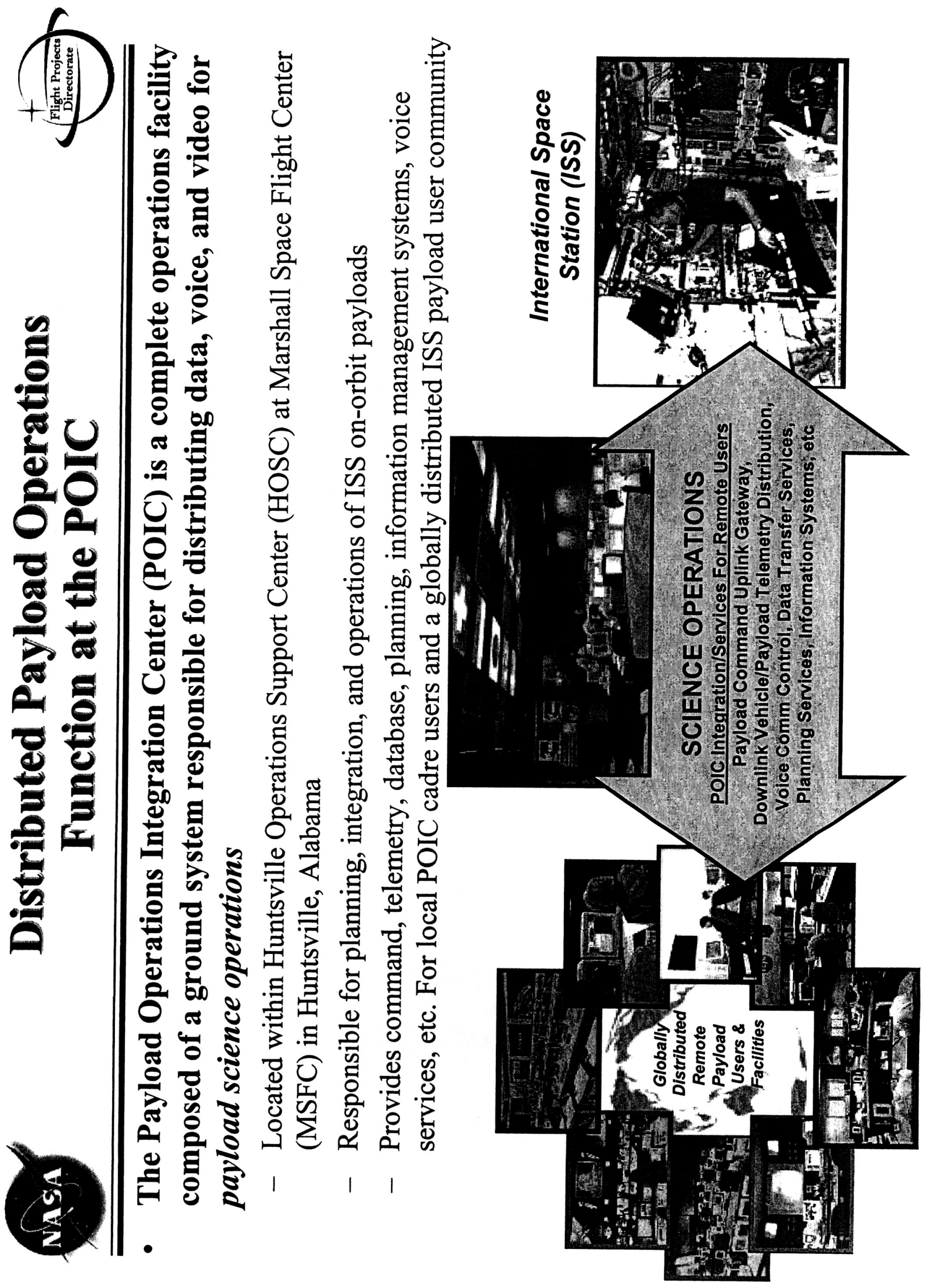




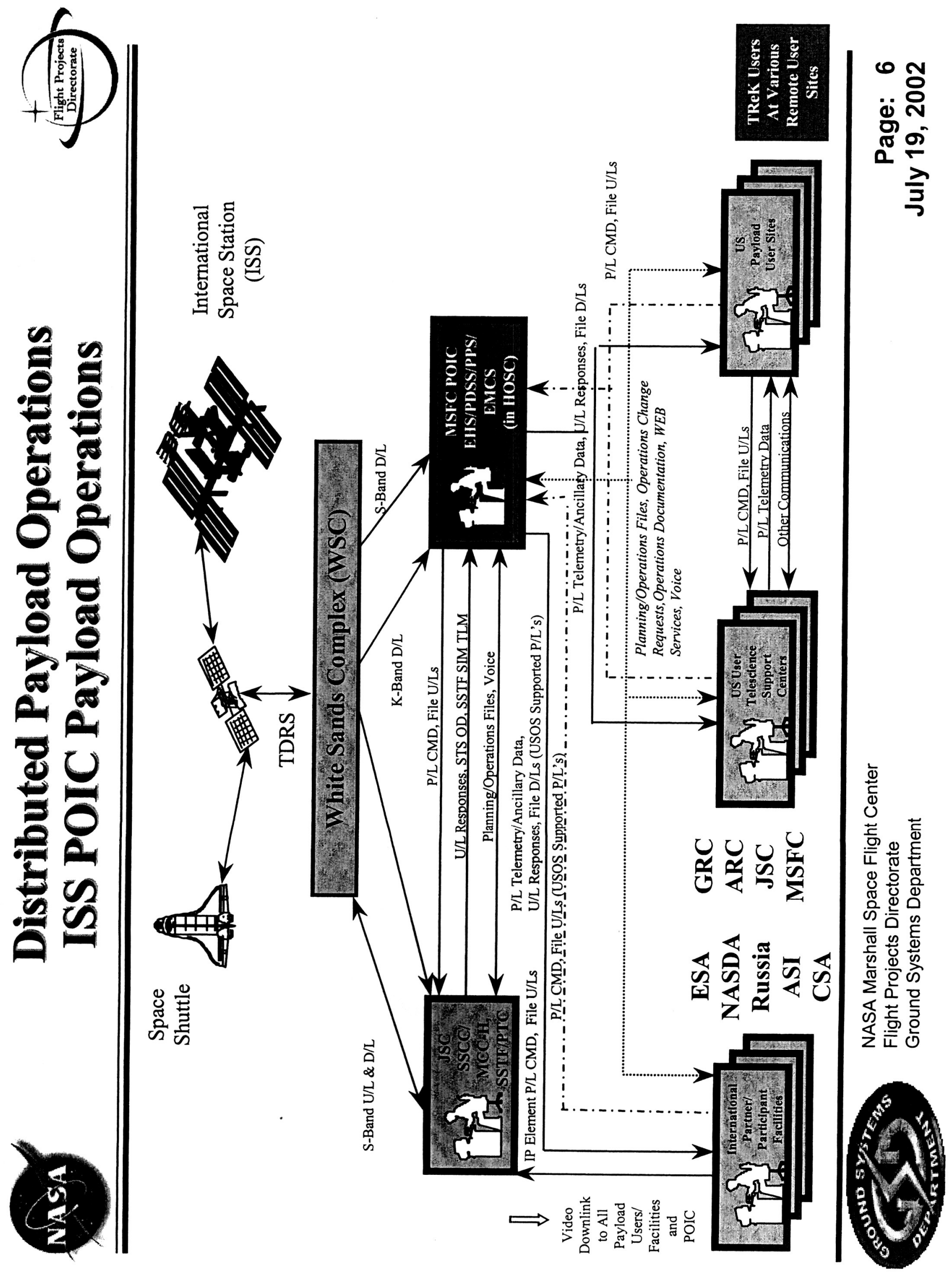




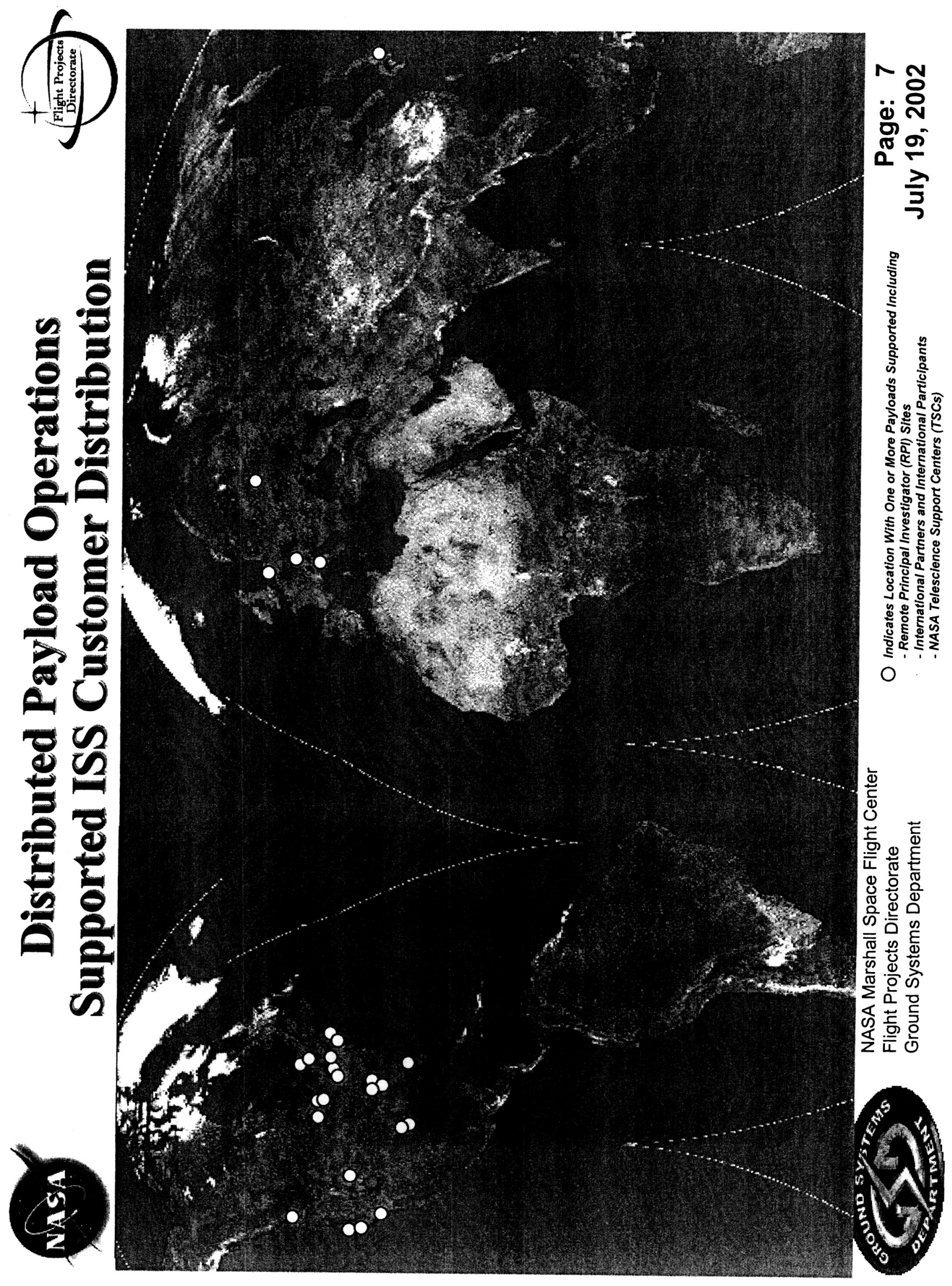




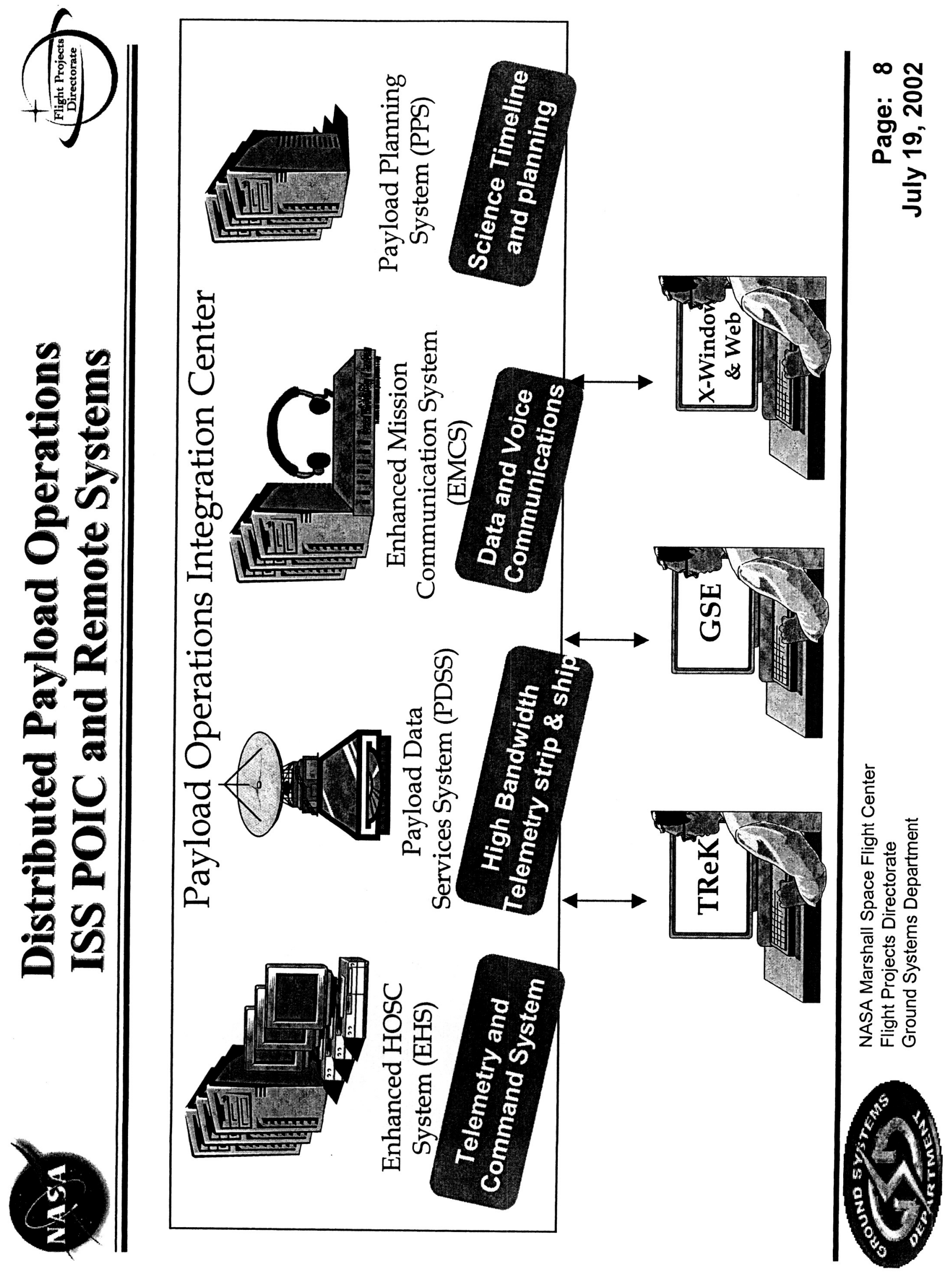




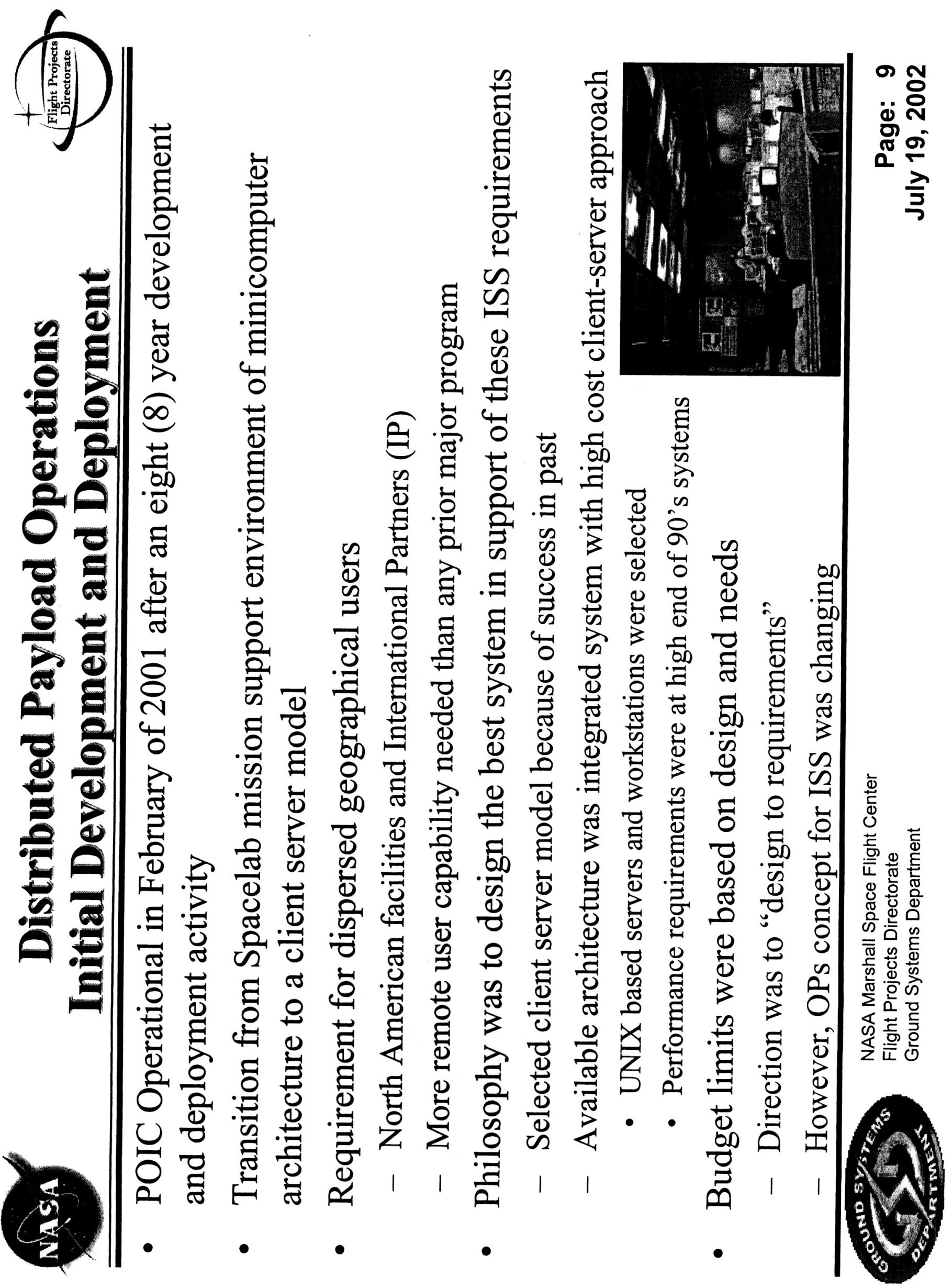




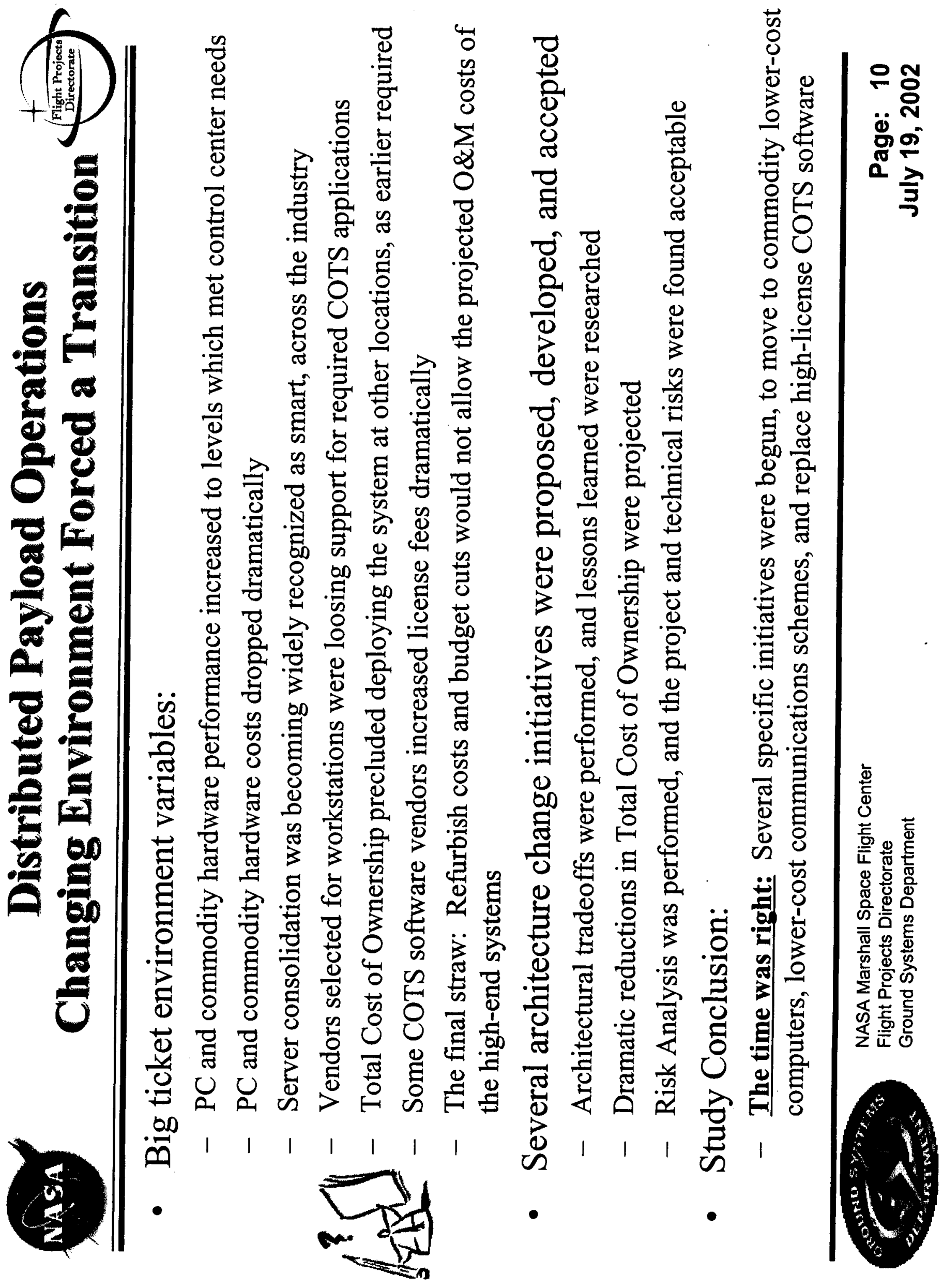




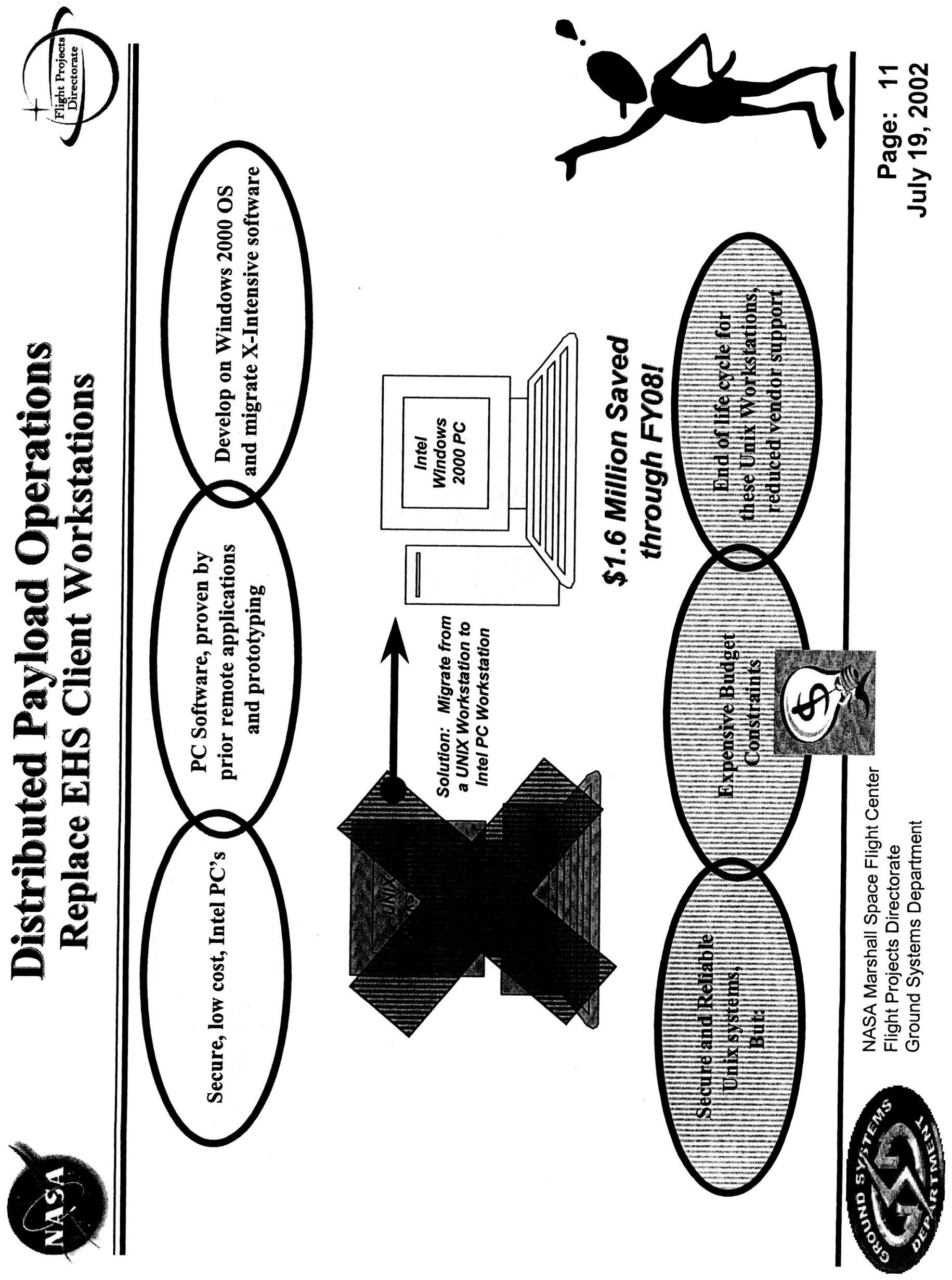




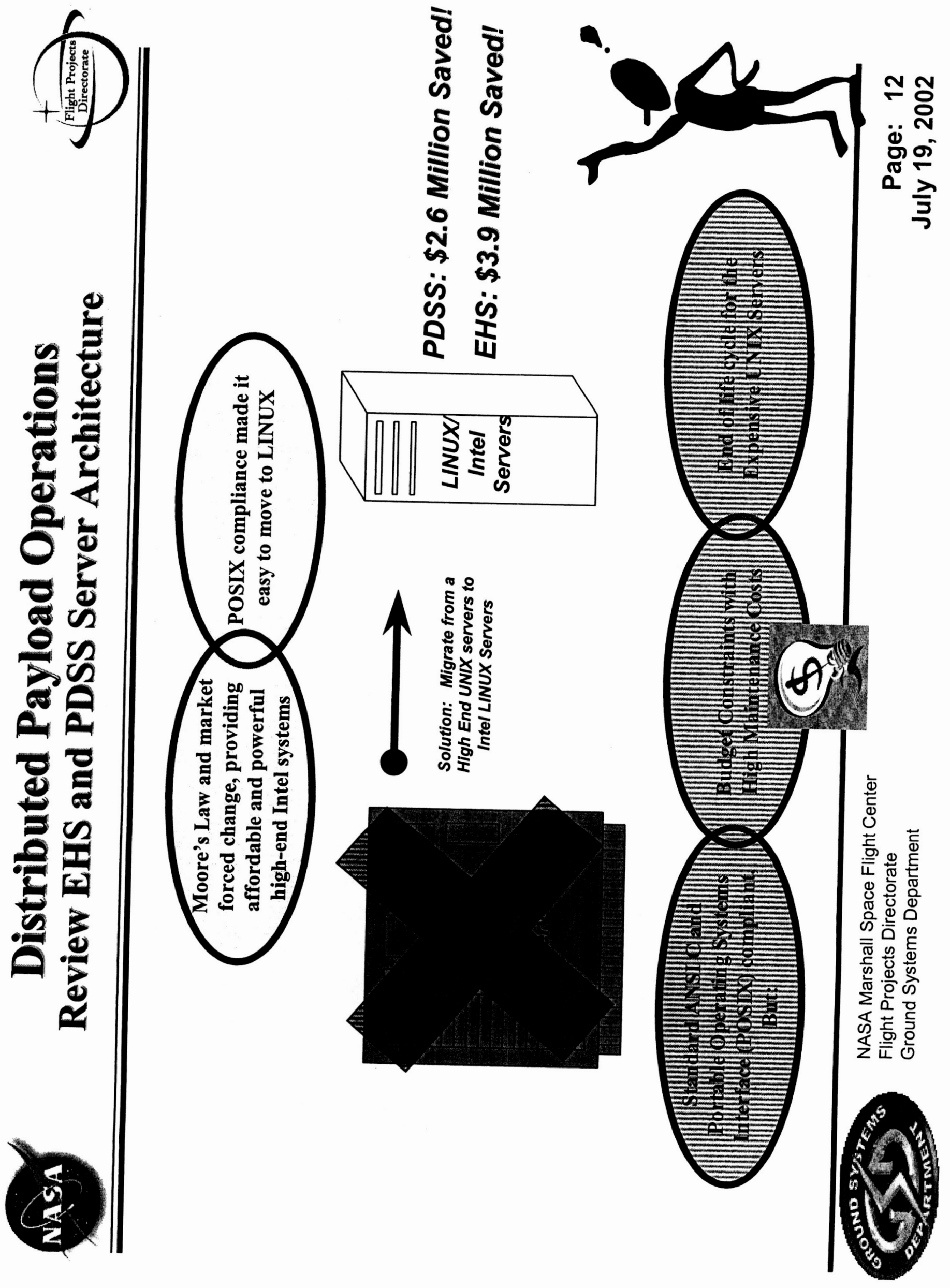




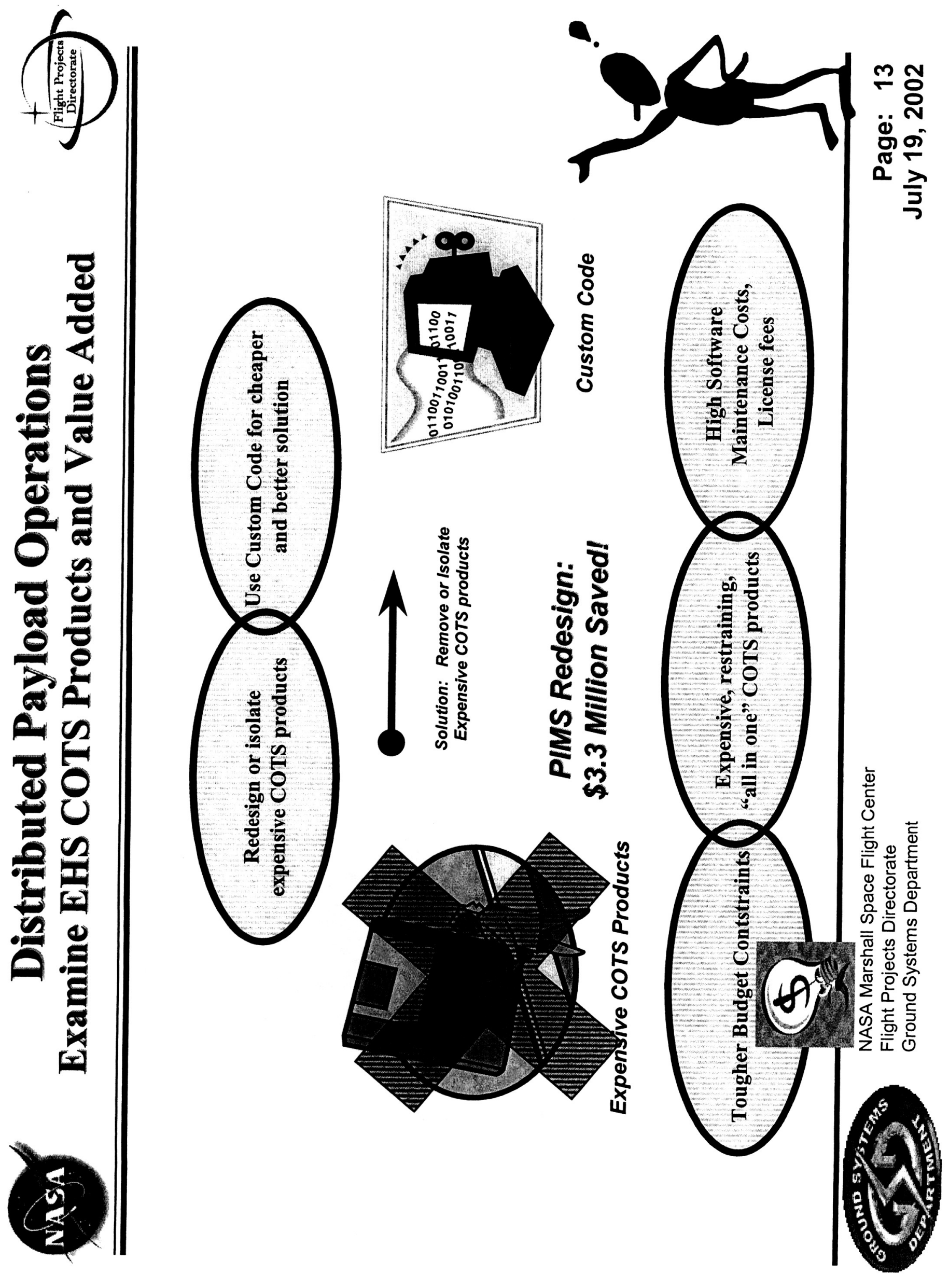




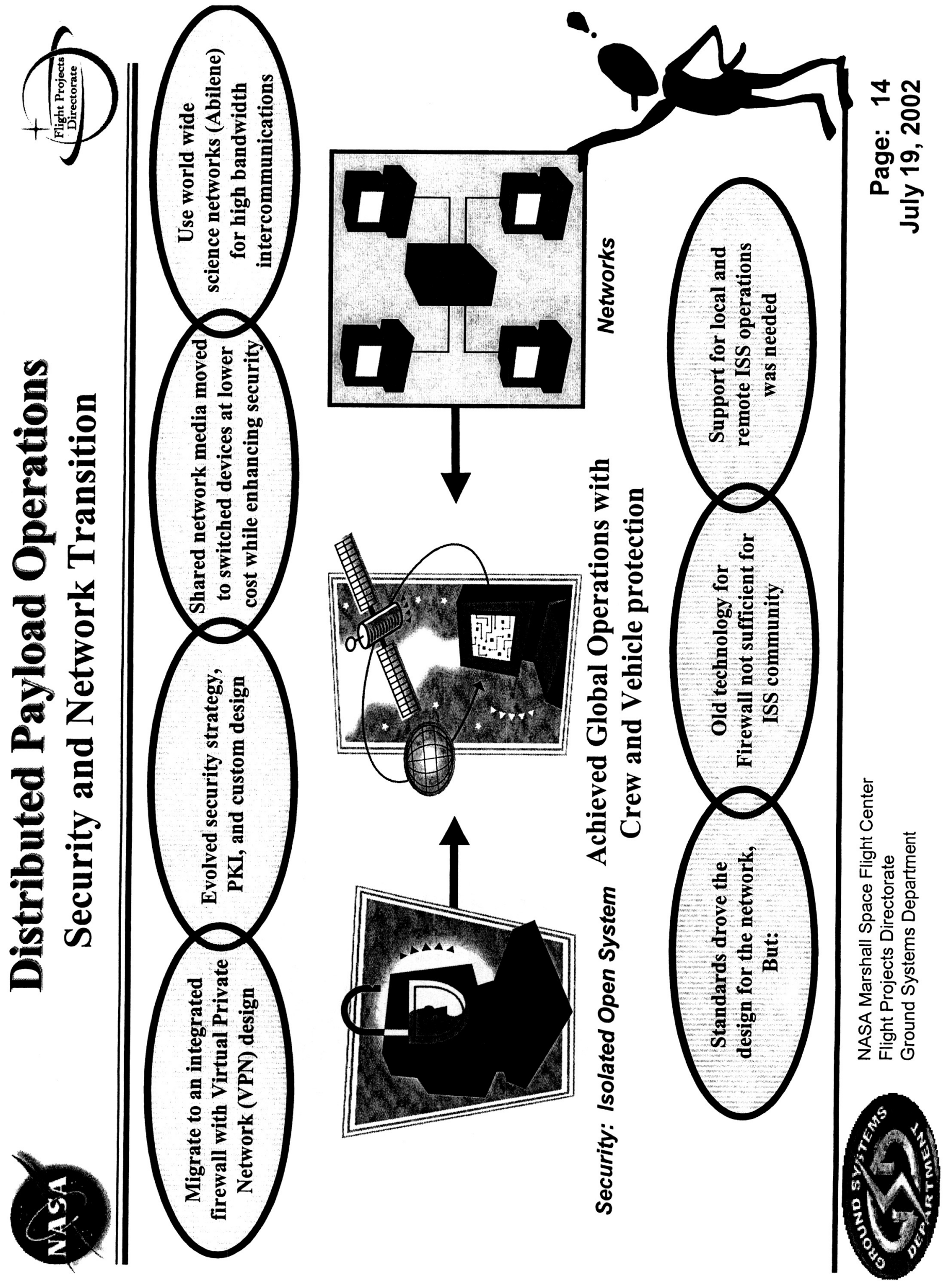




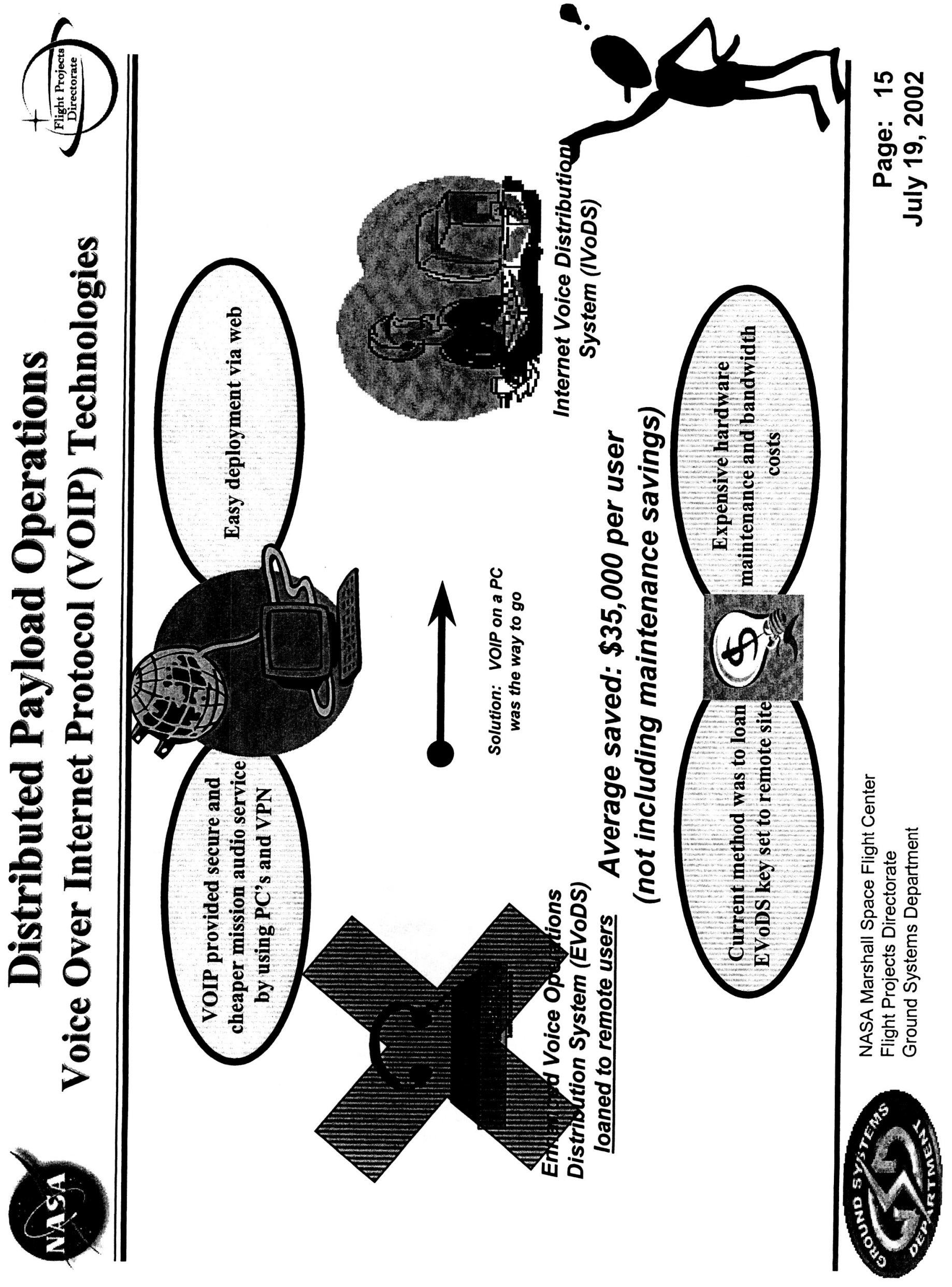




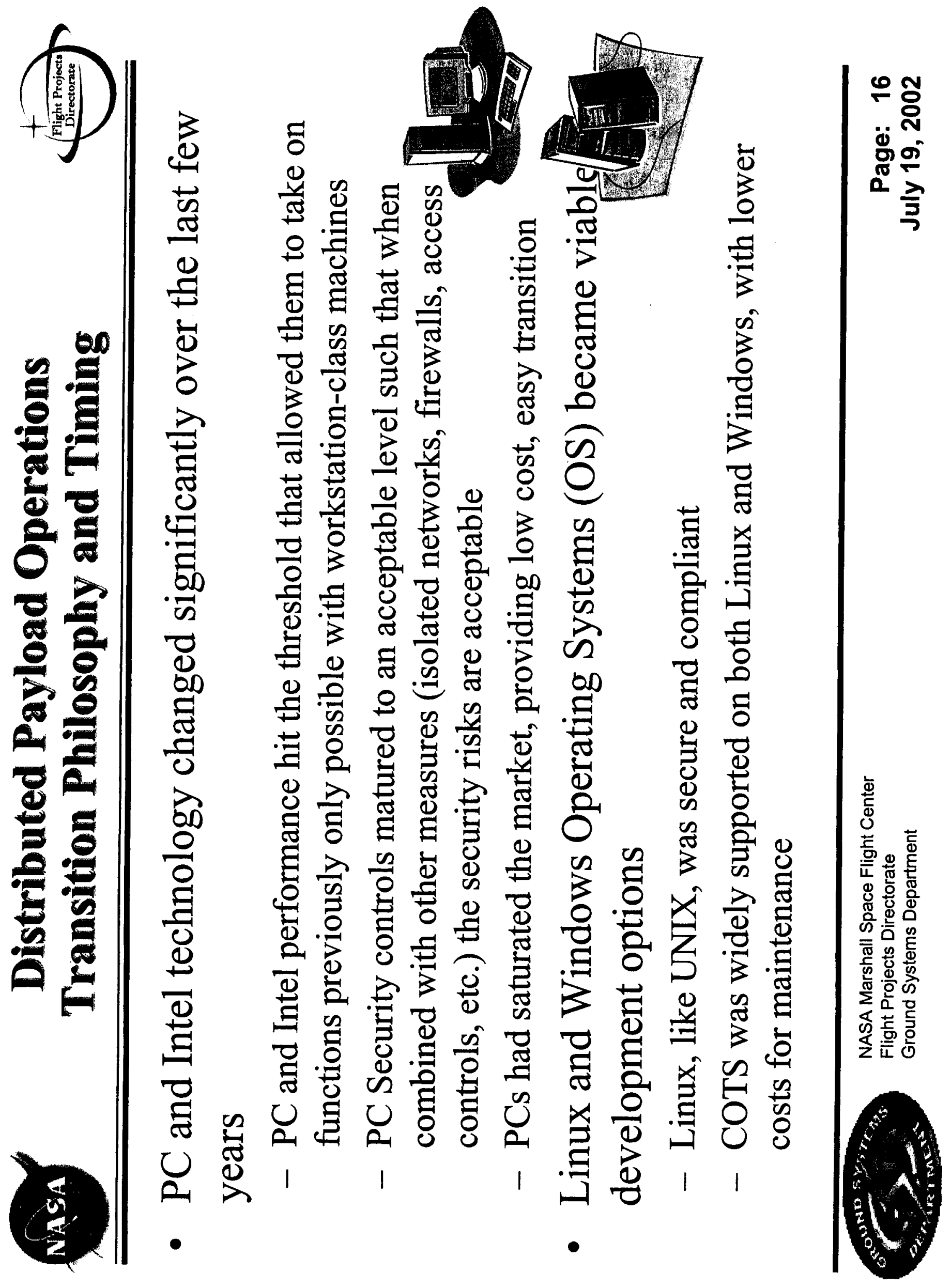




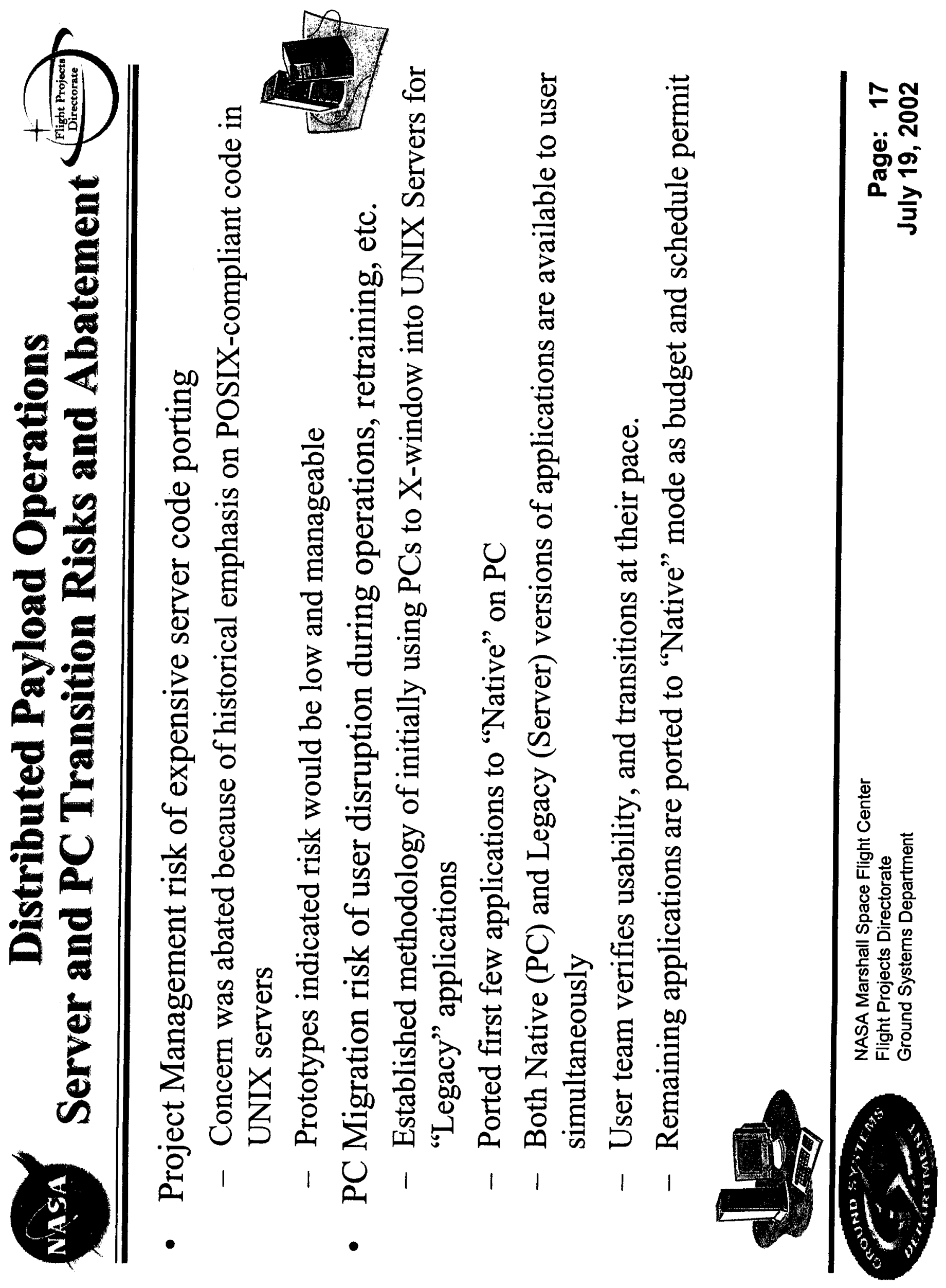




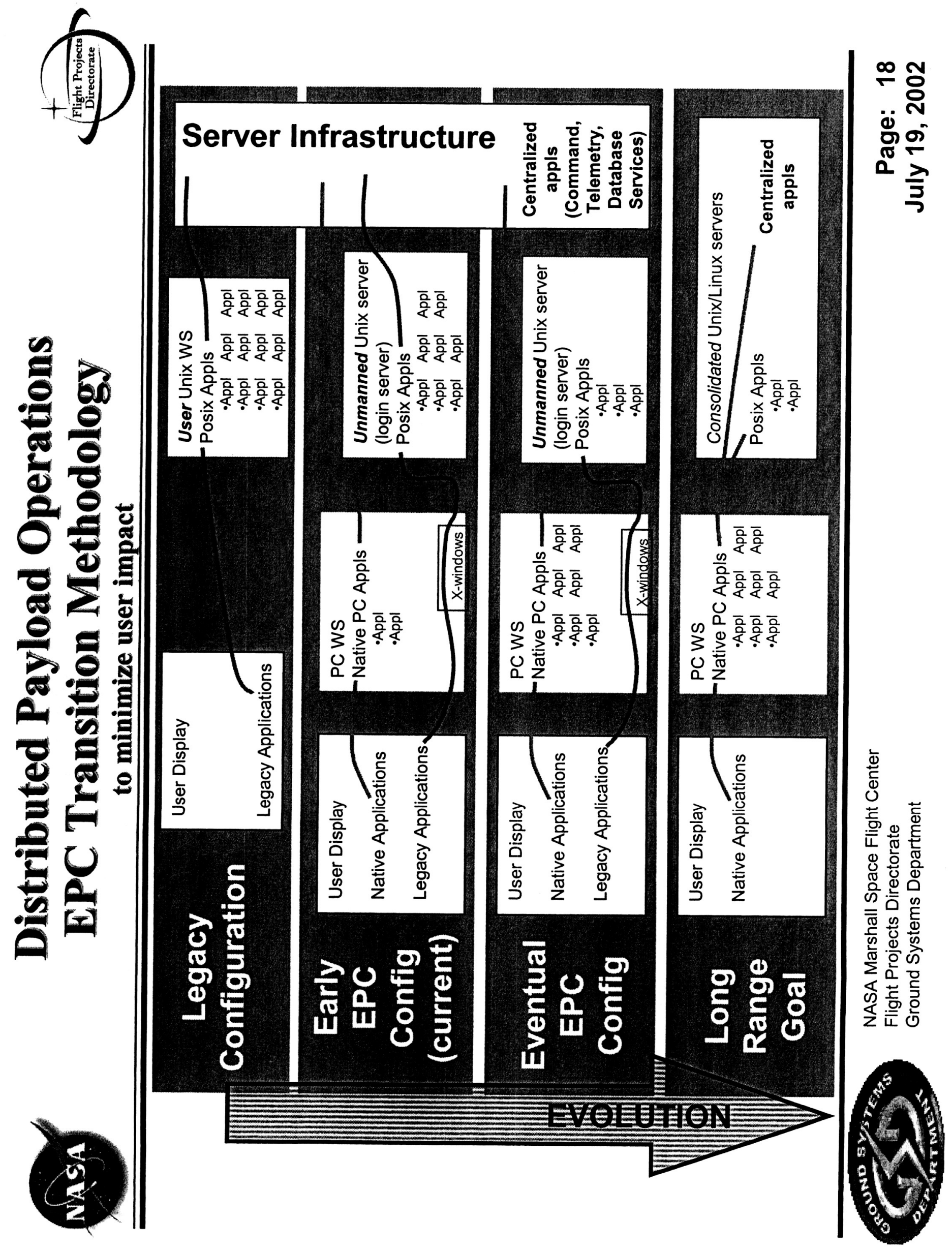




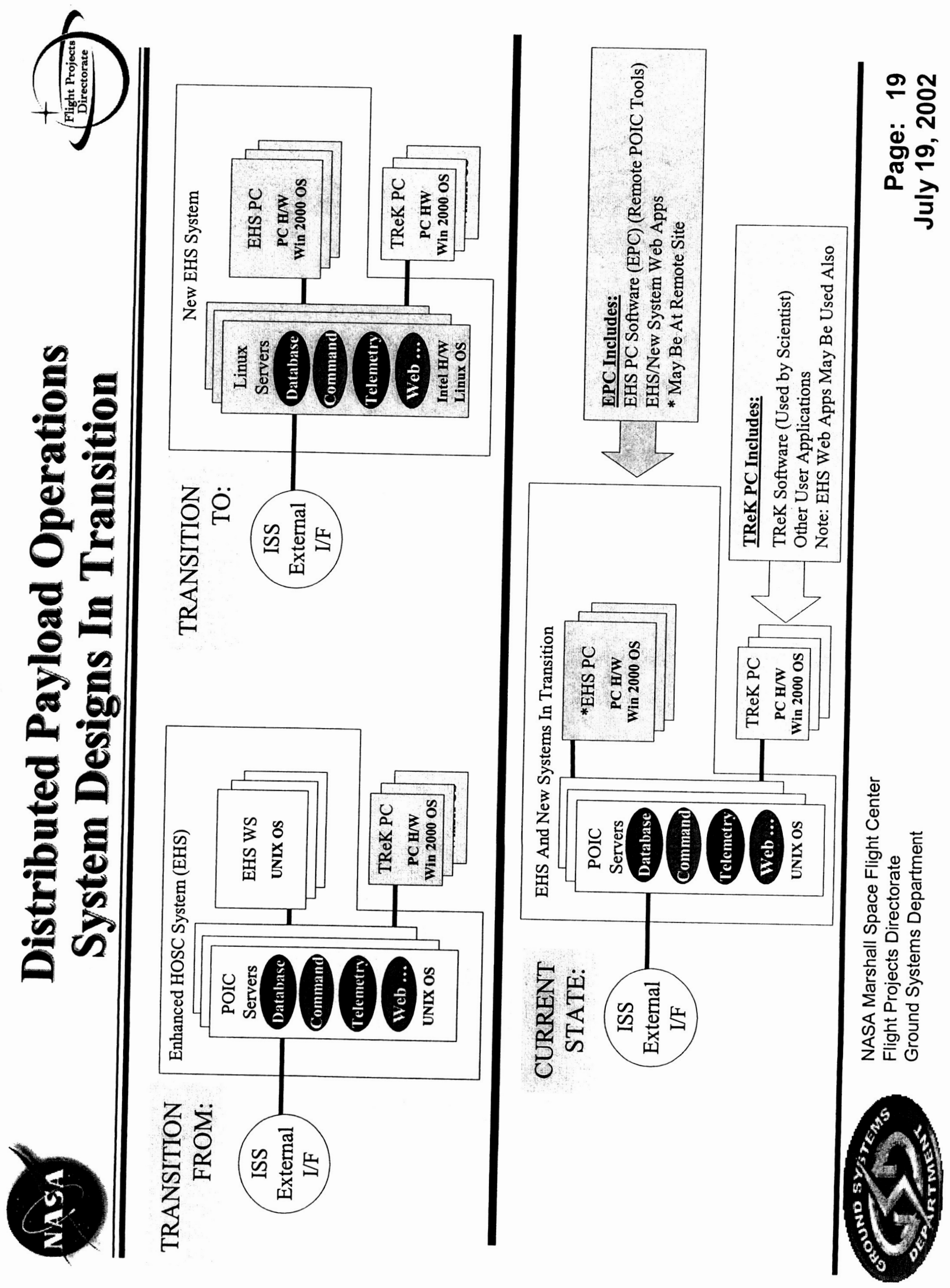




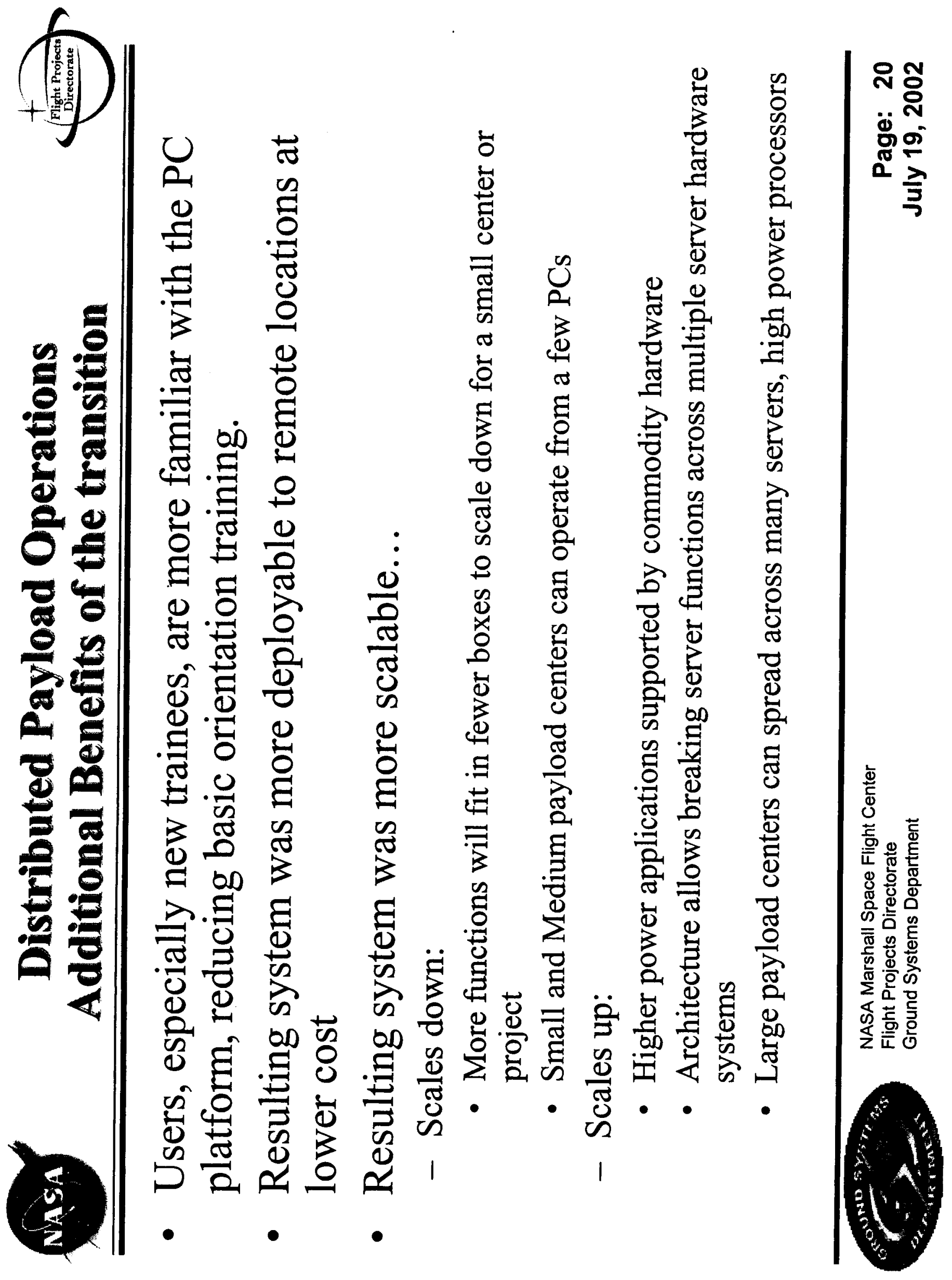




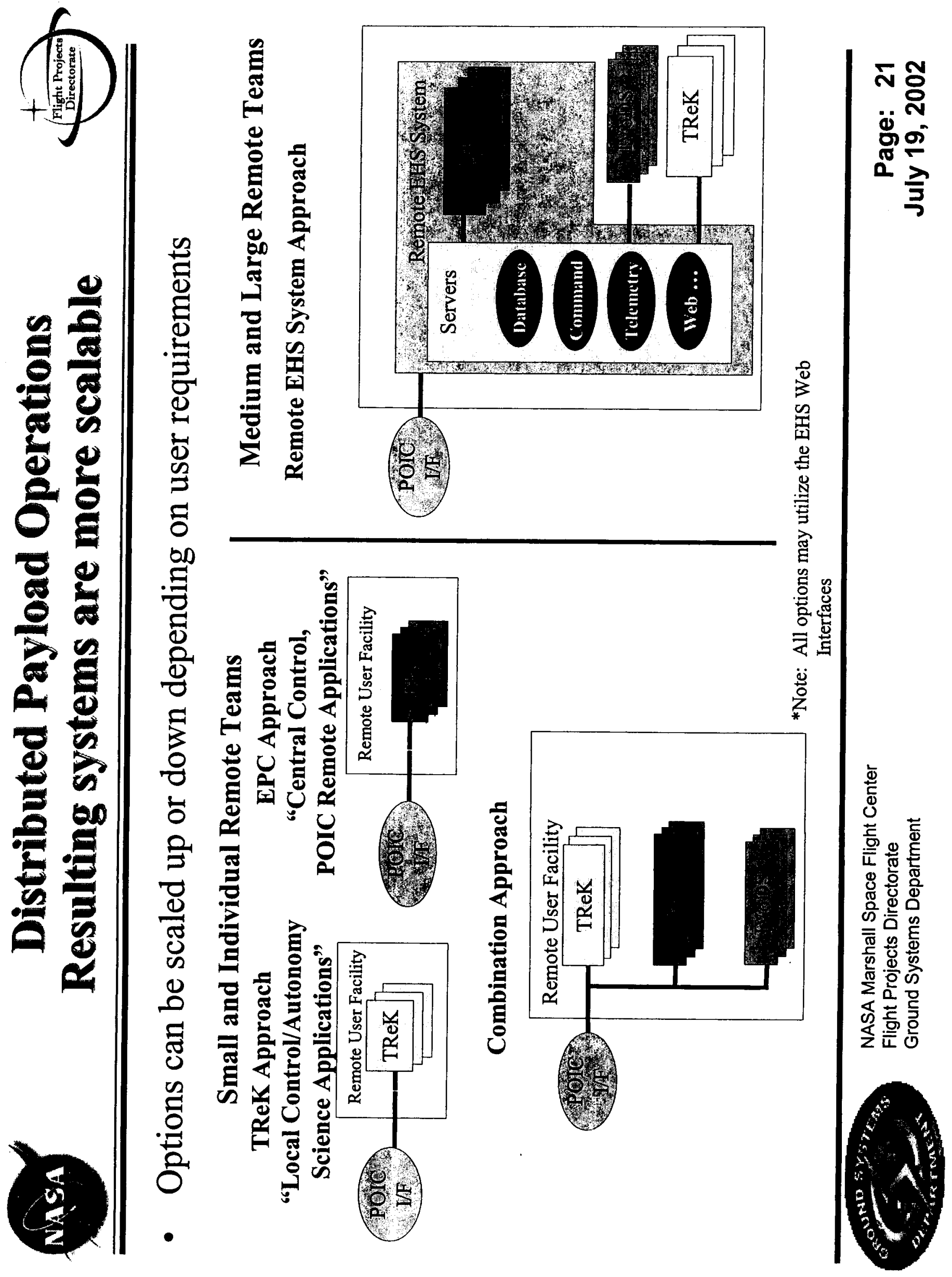



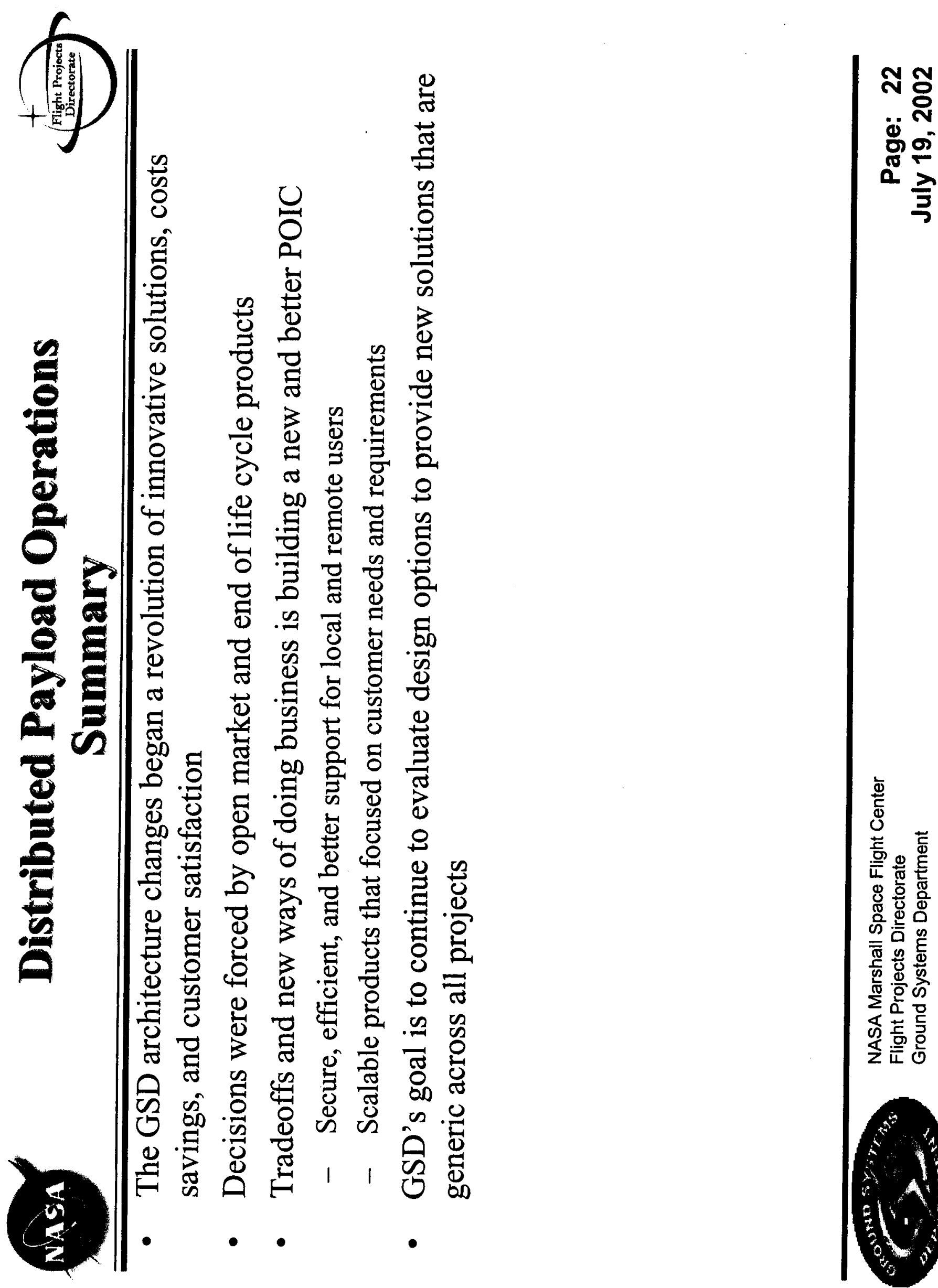


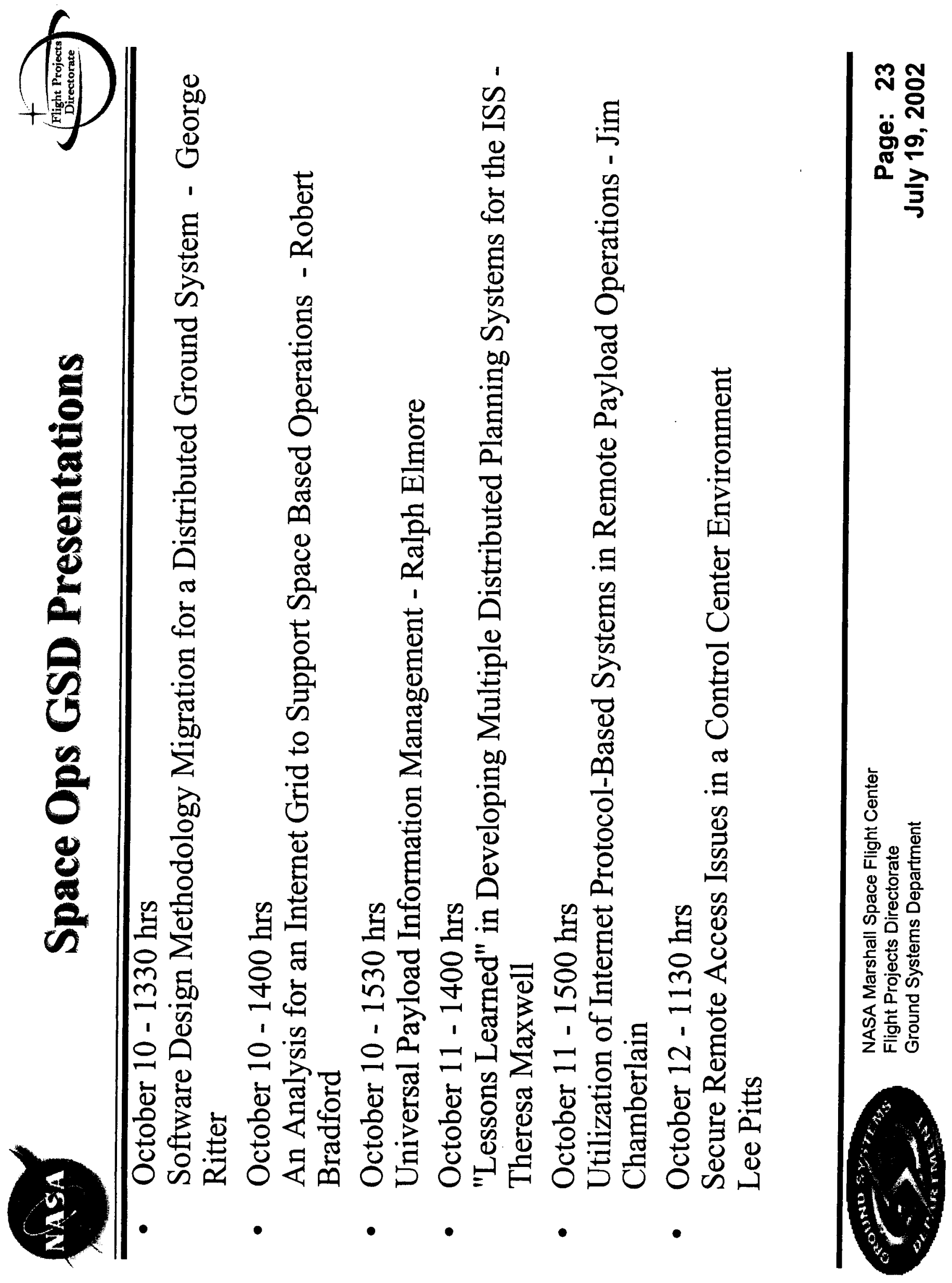




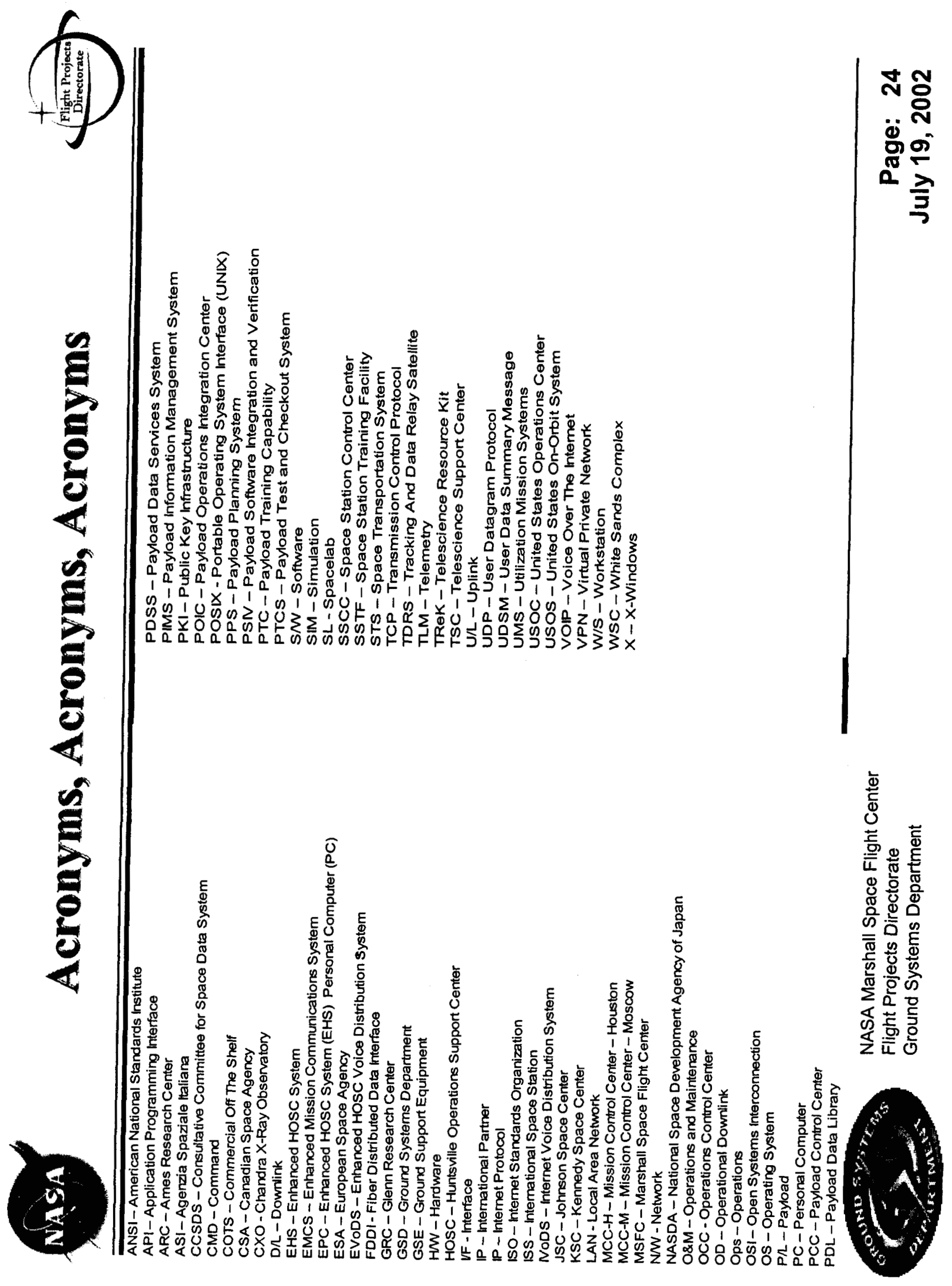

